\title{
Discretisations, Constraints and Diffeomorphisms in Quantum Gravity
}

\author{
Benjamin BAHR ${ }^{\dagger}$, Rodolfo GAMBINI ${ }^{\ddagger}$ and Jorge PULLIN $\S$ \\ $\dagger$ Department of Applied Mathematics and Theoretical Physics, University of Cambridge, \\ Wilberforce Road, Cambridge CB3 OWA, UK \\ E-mail:bab26@cam.ac.uk \\ $\ddagger$ Instituto de Física, Facultad de Ciencias, Universidad de la República, \\ Iguá 4225, CP 11400 Montevideo, Uruguay \\ E-mail: rgambini@fisica.edu.uy \\ $\S$ Department of Physics and Astronomy, Louisiana State University, \\ Baton Rouge, LA 70803-4001, USA \\ E-mail: pullin@lsu.edu
}

Received November 09, 2011, in final form December 31, 2011; Published online January 08, 2012 http://dx.doi.org/10.3842/SIGMA.2012.002

\begin{abstract}
In this review we discuss the interplay between discretization, constraint implementation, and diffeomorphism symmetry in Loop Quantum Gravity and Spin Foam models. To this end we review the Consistent Discretizations approach, which is an application of the master constraint program to construct the physical Hilbert space of the canonical theory, as well as the Perfect Actions approach, which aims at finding a path integral measure with the correct symmetry behavior under diffeomorphisms.
\end{abstract}

Key words: quantum gravity; diffeomorphisms; constraints; consistent discretizations; gauge symmetries; perfect actions; renormalization

2010 Mathematics Subject Classification: 37M99; 70H45; 81T17; 82B28; 83C27; 83C45

\section{Introduction}

The dynamics of General Relativity is governed by the Einstein-Hilbert action (together with the Gibbons-Hawking-York boundary term)

$$
S_{\mathrm{EH}}\left[g_{\mu \nu}\right]=\frac{1}{8 \pi} \int_{M} d^{4} x \sqrt{|\operatorname{det} g|}\left(\Lambda-\frac{1}{2} R\right)-\frac{1}{8 \pi} \int_{\partial M} d^{3} x \sqrt{\operatorname{det} h} K .
$$

The invariance of the action (1.1) under space-time diffeomorphisms (leaving the boundary invariant) implies a large redundancy on the set of solutions, so that two solutions are to be physically equivalent if they differ by such a diffeomorphism (Einstein's hole argument [1]). The diffeomorphism group therefore arises as gauge symmetry group of the theory.

Furthermore, unlike for usual gauge theories of connections where gauge transformations arise as local bundle automorphisms, the diffeomorphism symmetry is deeply intertwined with the dynamics of GR: The action (1.1) is the only diffeomorphism-invariant action of a metric which leads to second order equations of motion [2]. The symmetry requirement and the prescribed degrees of freedom therefore completely determine the theory.

\footnotetext{
*This paper is a contribution to the Special Issue "Loop Quantum Gravity and Cosmology". The full collection is available at http://www.emis.de/journals/SIGMA/LQGC.html
} 
On the canonical level the presence of a gauge symmetry results in first class constraints, forming the so-called Dirac algebra. The Hamiltonian is a linear combination of constraints, which generate both the dynamics and space-time diffeomorphisms [3].

The two main routes for quantizing GR are either taken via the path-integral formalism, where the transition amplitudes between states $\left|h_{a b}\right\rangle$ on initial and final Cauchy surfaces $\Sigma^{i, f}$ is given $b^{1}$

$$
\left\langle h_{a b}^{f} \mid h_{c d}^{i}\right\rangle=\int_{g \mid \Sigma^{i, f}=h^{i, f}} \mathcal{D} g_{\mu \nu} e^{\frac{i}{\hbar} S_{\mathrm{EH}}\left[g_{\mu \nu}\right]} .
$$

Canonical quantization of GR [5] on the other hand relies on a definition of a state space for the boundary metrics $|\psi\rangle$ whose evolution is governed by the Wheeler-DeWitt-equation [6]

$$
\hat{H}|\psi\rangle=0 .
$$

Demanding that the gauge symmetry of (1.1) is also realized in the quantum theory either manifests itself in the condition that the path integral measure in (1.2) is also invariant under diffeomorphisms, or that the Dirac algebra is faithfully represented on the state space of the canonical quantum theory. Attempts to make sense of either (1.2) or (1.3) very often rely on discretization of space-time on one way or the other, and since the interplay of discretization and space-time diffeomorphisms is highly nontrivial, this has been a central, and up to today unsolved issue in either attempt of formulating a theory of quantum gravity.

Out of the various proposals for a quantum gravity theory based on discretizations ${ }^{2}$, we concentrate in this article on Loop Quantum Gravity [11] and the closely related Spin Foam approach (see [12] for a review and literature), which can be seen as the path-integral version of canonical LQG (see e.g. the discussion in [13]).

\subsection{Canonical approach}

LQG is a canonical approach, in which the kinematical Hilbert space is well-understood, and the states of which can be written as a generalization of Penrose's Spin-Network Functions [14]. Although the formalism is inherently continuous, the states carry many discrete features of lattice gauge theories, which rests on the fact that one demands Wilson lines to become observables. The constraints separate into (spatial) diffeomorphism- and Hamiltonian constraints. While the finite action of the spatial diffeomorphisms can be naturally defined as unitary operators, the Hamiltonian constraints exist as quantizations of approximate expressions of the continuum constraints, regularized on an irregular lattice. It is known that the regularized constraints do not close, so that the algebra contains anomalies, whenever the operators are defined on fixed graphs which are not changed by the action of the operators [15]. If defined in a graphchanging way, the commutator is well-defined, even in the limit of the regulator going to zero in a controlled way [16]. However, the choice of operator topologies to choose from in order for the limit to exist is nontrivial [17], and the resulting Hamiltonian operators commute [18]. Since they commute on diffeomorphism-invariant states, the constraint algebra is satisfied in that sense. Furthermore, however, the discretization itself is not unique, and the resulting ambiguities survive the continuum limit [19]. In the light of this, it is non-trivial to check whether the correct physics is encoded in the constraints.

Generically, the interaction of discretizations and constraints is intricate. Physical theories based on continuous variables are typically discretized for two different purposes: a) to turn the

\footnotetext{
${ }^{1}$ The case in which $\Sigma^{i}$ is empty is called the "no-boundary proposal" and in this case (1.2) is called the "wave-function of the universe" [4].

${ }^{2}$ To this end, see [7], also in particular the Causal Dynamical Triangulations approach [8], Causal Set Theory [9], and Quantum Graphity [10].
} 
differential equations of the theory into difference equations in order to solve them numerically; b) to regularize and quantize the theory, as in lattice gauge theory. When the equations of the theory describe a free time evolution the main concern when discretizing is to achieve long term numerical stability. When the theories have constraints among their variables in addition to evolution equations the situation complicates. Typically the constraints satisfy certain conditions. Examples of such conditions are constraints that structure themselves into Poisson algebras in canonically formulated theories. Moreover constraints have to hold for all times, which implies that they need to be preserved by the evolution equations. The problem is that if one simply discretizes constraints and evolution equations these conditions generically get violated. At an operational level, to prove that constraints form an algebra or that they are preserved upon evolution one uses repeatedly the Leibniz property for derivatives, which fails to hold for discrete versions of derivatives. At a more profound level, constraints are associated with symmetries of theories that generically get broken by the discretization. If one insists in using the discrete equations, one is faced with the fact that generically they are inconsistent: they cannot be solved simultaneously. This phenomenon is well known in numerical relativity, where people proceed by ignoring some of the equations and solving the rest in the hope that the ignored equations, when evaluated on the solution, will be non-zero but small. Some algorithms can actually achieve this for long enough evolutions and that is the state of the art of that field.

These problems are pervasive even in simple theories. People tend to get misled by the success of lattice gauge theory without realizing that a careful choice of staggered discretization is used in order to preserve Gauss' law. In fact one of the central properties of lattice gauge theories is that it provides a gauge invariant regularization, i.e. it does not break the symmetries of the theory. To give some perspective, it is worthwhile noting that even a relatively simple theory like Maxwell theory has to be discretized judiciously. Attempts to integrate Maxwell's equations numerically ran into significant problems until Yee [20], in a landmark paper, introduced a staggered discretization that preserves Gauss' law.

Going to the case of interest, that of general relativity and other diffeomorphism invariant theory, we currently do not know of any discretization scheme that would preserve the constraints of the theory. So we are, effectively, where people were with respect to Maxwell's theory before Yee's algorithm. Attempts have been made to define a discrete calculus, but up to present successful implementations only are possible in the linearized theory.

In Section 2, we will discuss these issues in more details for models that share important features with General Relativity, and demonstrate the consistent and uniform discretization approach, in order to deal with the aforementioned problems.

\subsection{Covariant approach}

On the covariant level, a very convenient and geometric way of discretizing GR is Regge Calculus, in which the metric variables are discretized on a triangulation, i.e. a separation of space-time into discrete building blocks. Although evolved from a different perspective, the Spin Foam approaches can be seen as providing a path integral for Regge Calculus [21] in connection-, rather than metric variables.

It is known that, generically, discretizing mechanical systems which possess a reparametrization-invariance breaks this invariance [22] (see also the discussion in [23]). There has been an intensive discussion in the literature about whether some invariance akin to diffeomorphisms exist in the case of Regge Calculus [24, 25]. While it is well-known that some very specific solutions, describing a flat metric, can be related via continuous gauge transformations [26, 27, 28], it was also demonstrated in [29] that this symmetry is broken whenever solutions describe geometries with curvature, and the breaking mechanism is completely analogous to what happens in mechanical systems. Since the current Spin Foam models, at least in the semiclassical limit 
of large quantum numbers, reproduce Regge Calculus [30], it has to be expected that the same breaking of diffeomorphism invariance also appears in the path integral measure.

In Section 3 we will discuss the mechanism of how diffeomorphism-symmetry is broken in Regge Calculus, and describe an attempt to regain it by "improving" the Regge action. We will show how one can attempt to construct a discrete action with the correct symmetries by a coarse graining procedure, resembling a Wilsonian renormalization group flow. We will also demonstrate how a similar procedure works for the toy example of the (an-)harmonic oscillator, closing the section with remarks on consequences for renormalization in Spin Foam models. Also, it will be argued how the symmetry breaking the path integral measure will account for divergencies in the "sum over triangulations".

Although LQG and Spin Foam models agree well on the kinematical level, the connection of their dynamics is still not understood perfectly [13,31]. Therefore it is hard to argue that the methods described in this article exactly correspond to each other. Still, they are both attempts to solve problems that arise due to the introduction of a discretization. Also, in [32] it was shown that, for a theory of gravity discretized on a triangulation, finding an action with the right symmetries, and finding constraints satisfying the Dirac algebra (and therefore generating deformations of the triangulated Cauchy surface), are equivalent. For a toy model, this was shown to be true for the quantum case, i.e. a path integral measure with the right kind of symmetries, and the propagator being the correct projector onto the physical Hilbert space, this was shown in [33]. In this sense, the problems on the canonical and the covariant side are directly related to each other, and the two approaches described in this article can be viewed as addressing the problem from different angles.

It is important to note that the breaking of gauge symmetry in the current approaches to Quantum Gravity arise due to the intricate relationship between space-time diffeomorphisms and the discretization involved. Although it is widely expected that any Quantum Gravity theory should predict a fundamental space-time discreteness due to the presence of a minimal length scale, this does not necessarily require a breaking of diffeomorphism symmetry, but is rather a statement about the discreteness of the spectrum of geometric operators measuring lengths, areas, etc. [34].

\section{Consistent discretisation}

Consistent discretisations [35] were an attempt to discretise theories like general relativity in which the resulting discretised equations were algebraically consistent, that is, they could be solved simultaneously. Notice that this is not what happens if one just takes the Einstein equations and discretises them. The Einstein equations divide into constraints that hold at every instant of time and evolution equations that evolve the variables. It turns out that if one evolves the variables with the discrete equations, at the end of the evolution the variables fail to satisfy the constraints. The resulting equations are therefore inconsistent. This is well known, for instance, in numerical relativity. The way consistent discretisations will make the equations consistent is by determining the values of the Lagrange multipliers. That is, the latter stop being free variables in the discrete theory. We will see that a problem arises in that the equations determining the Lagrange multipliers are polynomials and therefore it is not guaranteed that the multipliers remain real. And since one loses control of their values, one cannot guarantee that the resulting discretisation will approximate well the continuum theory. This problem is addressed by the Uniform Discretisations, which we discuss in Section 2.2.

Our goal would be to have a canonical quantization of a system in question using a discretization. Let us outline the steps we would like to see carried out:

- We discretize the theory to be analyzed. 
- We study the resulting discrete theory at a classical level (in particular making sure it is a well defined theory).

- We canonically quantize such a theory.

- We either take the continuum limit of the resulting quantum theory or show that it does not exist.

We are particularly interested in studying systems with common features to those of general relativity: invariance under diffeomorphisms, the presence of first class constraints, the theory being totally constrained, among others.

If the starting theory is invariant under diffeomorphisms, the discretization inevitably will break that symmetry. The discrete theory has less gauge symmetries and therefore more degrees of freedom than the continuum one. When one works in a Hamiltonian picture, space and time have asymmetrical roles, so the breakage of diffeomorphism symmetry manifests itself differently in space than in time. If one only discretizes in time, the breaking of the invariance leads to the corresponding constraints being absent in the discrete theory and the corresponding Lagrange multipliers get determined. We will see an example soon. If one discretizes spatially only, the breakage translates itself in the constraints associated with spatial diffeomorphisms becoming second class and therefore fail to be generators of symmetries.

\subsection{Systems with discrete time}

There is an alternative way to construct a discretized version of a theory that instead of simply discretizing evolution equations and constraints starts by discretizing the action of the theory. One subsequently works out the equations of motion of such action. Unless pathologies arise, the resulting equations are consistent, as they are simply the equations of motion of an action. We call this approach consistent discretizations and refer the reader to [35] for more details. Let us discuss how this works in the case of a simple mechanical system.

Suppose one has a mechanical system with Lagrangian $\hat{L}(q, \dot{q})$ where generically $q$ and $\dot{q}$ are $N$ component vectors, representing a system with $N$ degrees of freedom. We discretize time in equal intervals $t_{n+1}-t_{n}=\varepsilon$. We denote the coordinates at $t_{n}$ as $q_{n}$ For simplicity we use a first order approximation for derivatives $\dot{q}=\left(q_{n+1}-q_{n}\right) / \varepsilon$. One can choose other approximations for the derivatives that may perform better numerically, but here for simplicity we keep the first order one. We define a Lagrangian for the discrete theory as

$$
L(n, n+1) \equiv L\left(q_{n}, q_{n+1}\right) \equiv \varepsilon \hat{L}(q, \dot{q}) .
$$

In terms of this Lagrangian the action can be written as

$$
S=\sum_{n=0}^{N} L\left(q_{n}, q_{n+1}\right)
$$

and from it we can derive the equations of motion

$$
\frac{\partial S}{\partial q_{n}}=\frac{\partial L\left(q_{n-1}, q_{n}\right)}{\partial q_{n}}+\frac{\partial L\left(q_{n}, q_{n+1}\right)}{\partial q_{n}}=0 .
$$

Notice that in the discrete theory the Lagrangian is a function of $q_{n}$ at the $n$-th level and of $q_{n+1}$ at the next level (or equivalently $q_{n}$ and $q_{n-1}$ ).

In order to formulate the theory canonically we need to define conjugate momenta. At first this may appear surprising. If there is no variable $\dot{q}$ anymore, how could there be a canonical momentum? In the continuum the Lagrangian is a function of the cotangent bundle of the 
configuration space $C$. In the discrete theory the Lagrangian is a function of $C \times C$. One can change from the coordinatization $\left(q_{n}, q_{n+1}\right)$ to $\left(q_{n}, p_{n}\right)$ defining the momentum. For that purpose it is useful to relate the continuum and discrete derivatives of the Lagrangians

$$
\begin{aligned}
& \frac{\partial L(n, n+1)}{\partial q_{n+1}}=\frac{\partial \hat{L}}{\partial \dot{q}}, \\
& \frac{\partial L(n, n+1)}{\partial q_{n}}=\varepsilon \frac{\partial \hat{L}}{\partial q}-\frac{\partial \hat{L}}{\partial \dot{q}} .
\end{aligned}
$$

The Lagrange equations in the continuum can be written as

$$
\frac{d p}{d t}=\frac{\partial \hat{L}}{\partial q}
$$

To discretize this last expression we make the substitution

$$
\dot{p} \rightarrow \frac{p_{n+1}-p_{n}}{\varepsilon}
$$

and use (2.2), (2.3) to get

$$
\begin{aligned}
\frac{p_{n+1}-p_{n}}{\varepsilon} & =\frac{\partial \hat{L}}{\partial q}=\frac{1}{\varepsilon}\left[\frac{\partial \hat{L}}{\partial \dot{q}}+\frac{\partial L(n, n+1)}{\partial q_{n}}\right] \\
& =\frac{1}{\varepsilon}\left[\frac{\partial L(n, n+1)}{\partial q_{n+1}}+\frac{\partial L(n, n+1)}{\partial q_{n}}\right]=\frac{1}{\varepsilon}\left[p_{n+1}+\frac{\partial L(n, n+1)}{\partial q_{n}}\right] .
\end{aligned}
$$

Then

$$
p_{n}=-\frac{\partial L(n, n+1)}{\partial q_{n}} .
$$

The resulting discrete Lagrange equations are

$$
p_{n+1}=\frac{\partial L\left(q_{n}, q_{n+1}\right)}{\partial q_{n+1}}, \quad p_{n}=-\frac{\partial L\left(q_{n}, q_{n+1}\right)}{\partial q_{n}} .
$$

It is worthwhile pointing out that these equations define a type 1 canonical transformation from the variables $\left(q_{n}, p_{n}\right)$ to $\left(q_{n+1}, p_{n+1}\right)$. This transformation is canonical, in the sense that it preserves the symplectic structure

$$
\left\{q_{n}, p_{n}\right\}=\left\{q_{n+1}, p_{n+1}\right\}=1 \text {. }
$$

In order to make contact with the continuum theory, we point out that if one takes as discrete Lagrangian the Jacobi principal function $S(q, Q, \varepsilon)$ then the discrete evolution given by (2.4) will correspond to the evolution of the continuous system for times $t=n \varepsilon$. This is an alternative view on discrete Lagrangians: they are approximants to the function $S(q, Q, \varepsilon)$. This is closely related to the idea of a symplectic (also known as variational) integrator [22].

We note that there exists a discrete version of Noether's theorem. If the system has a global symmetry $\delta_{u} q_{n}=u \delta q_{n}$, then the discrete Lagrangian will be invariant up to a total difference

$$
\delta_{u} L=u\left(\frac{\partial L\left(q_{n}, q_{n+1}\right)}{\partial q_{n}} \delta q_{n}+\frac{\partial L\left(q_{n}, q_{n+1}\right)}{\partial q_{n+1}} \delta q_{n+1}\right)=u\left(B_{n+1}-B_{n}\right),
$$


and using the equation of motion (2.1), we get the conserved quantity

$$
C_{n}=-\frac{\partial L}{\partial q_{n}} \delta q_{n}+B_{n}
$$

(that is $C_{n+1}=C_{n}$ ).

If the action is invariant under a "local" transformation $\delta_{u} q_{n}=u(n) \delta q_{n}$, we have

$$
0=\delta_{u} S=\sum_{n} \frac{\partial S}{\partial q_{n}} \delta q_{n} u(n)
$$

and since the $u(n)$ are arbitrary, this means that $\frac{\partial S}{\partial q_{n}} \delta q_{n}=0$, that is

$$
\left(\frac{\partial}{\partial q_{n}}\left(L\left(q_{n-1}, q_{n}\right)+L\left(q_{n}, q_{n+1}\right)\right)\right) \delta q_{n}=0
$$

This implies that the equations of motion (2.1) will not be independent and therefore one cannot unambiguously solve $q_{n+1}$ in terms of $q_{n}$ and $q_{n-1}$. That is, the system will be singular. In particular the Legendre transform $q_{n+1} \rightarrow p_{n}=-\frac{\partial L_{\epsilon}}{\partial q_{n}}$ will be singular and there will be constraints in phase space. The framework to treat constrained systems of this sort parallels Dirac's method in the continuum and is described in the second reference of [35].

Let us conclude our classical discussion considering an example of a simple singular discrete system: a parameterized free particle whose continuum Lagrangian is $\tilde{L}=\frac{m}{2} \dot{x}^{2} / \dot{t}$, and we consider the following discretization

$$
L\left(x_{n}, t_{n}, x_{n+1}, t_{n+1}\right)=\frac{m}{2} \frac{\left(x_{n+1}-x_{n}\right)^{2}}{\left(t_{n+1}-t_{n}\right)} .
$$

This discretization inherits its singular nature from the continuum. In particular, when we compute the momenta $p_{n}^{x}=-\partial L / \partial x_{n}$ and $p_{n}^{t}=-\partial L / \partial t_{n}$ we see they are not independent but they satisfy the same relations as the continuum ones

$$
\phi=p_{n}^{t}+\frac{1}{2 m}\left(p_{n}^{x}\right)^{2}=0 .
$$

The "local" invariance of the Lagrangian is given by the transformations $\delta_{u} x_{n}=u(n) \delta x_{n}$, $\delta_{u} t_{n}=u(n) \delta t_{n}$, where

$$
\delta t_{n}=1, \quad \delta x_{n}=\frac{1}{2 m}\left(\frac{x_{n+1}-x_{n}}{t_{n+1}-t_{n}}+\frac{x_{n}-x_{n-1}}{t_{n}-t_{n-1}}\right),
$$

and this transformation satisfies (2.5).

In phase space the transformation is given by $\delta t_{n}=1$ and $\delta x_{n}=1 /(2 m)\left(p_{n}^{x}+p_{n-1}^{x}\right)$. Using the equations of motion we have that $p_{n-1}^{x}=p_{n}^{x}$ which implies

$$
\delta t_{n}=1, \quad \delta x_{n}=\frac{1}{m} p_{n}^{x} .
$$

This is the general transformation generated by the constraint $\phi$.

Let us briefly discuss how to quantize these discrete systems. The general treatment is discussed in [36]. The treatment is similar to ordinary quantum mechanics, but evolving in discrete intervals $\varepsilon$. The starting point is as in the continuum, one considers wavefunctions $\psi(q)$, defining the operators $\hat{q}$ and $\hat{p}$ as usual, and the canonical evolution is implemented through a 
unitary operator. That is, if we call $Q(q, p)$ and $P(q, p)$ to the solution of the classical equations of motion (2.4), we need a unitary operator $\hat{U}$ such that

$$
Q(\hat{q}, \hat{p})=\hat{U} \hat{q} \hat{U}^{\dagger}, \quad P(\hat{q}, \hat{p})=\hat{U} \hat{p} \hat{U}^{\dagger},
$$

which represent the discrete evolution equations in the Heisenberg representation.

In the position basis equations (2.6) are a set of differential equations that determine the matrix $U\left(q^{\prime}, q\right)$. In some cases one can show that the solution to such equations is $U\left(q^{\prime}, q\right)=$ $C \exp \frac{i}{\hbar} L_{\epsilon}\left(q, q^{\prime}\right)$ with $C$ a proportionality constant.

We illustrate the quantization with a simple example given by a particle in a potential. The Lagrangian is

$$
L\left(q_{n}, q_{n+1}\right)=m \frac{\left(q_{n+1}-q_{n}\right)^{2}}{2 \epsilon}-V\left(q_{n}\right) \epsilon
$$

the canonical momentum is given by $p_{n+1}=m\left(q_{n+1}-q_{n}\right) / \epsilon$, from which we can get $q_{n+1}=$ $p_{n+1} \epsilon / m+q_{n}$. The generating function is,

$$
F_{2}=p_{n+1} q_{n}+\frac{p_{n+1}^{2}}{2 m} \epsilon+V\left(q_{n+1}\right) \epsilon=p_{n+1} q_{n}+H\left(p_{n+1}, q_{n}\right),
$$

and the equations of motion derived from the corresponding canonical transformation are

$$
q_{n+1}=q_{n}+\frac{p_{n+1}}{m} \epsilon, \quad p_{n}=p_{n+1}+V^{\prime}\left(q_{n}\right) \epsilon,
$$

which can be solved for $p_{n+1}$ as

$$
q_{n+1}=q_{n}+\frac{p_{n}}{m} \epsilon-V^{\prime}\left(q_{n}\right) \frac{\epsilon^{2}}{m}, \quad p_{n+1}=p_{n}-V^{\prime}\left(q_{n}\right) \epsilon .
$$

To quantize the system we choose a polarization such that the wavefunctions are functions of the configuration variables, $\Psi\left(q_{n+1}\right)$. The canonical operators have the usual form. The evolution of the system is given by a unitary transformation, $p_{n+1}=U p_{n} U^{\dagger}, q_{n+1}=U q_{n} U^{\dagger}$ and the unitary operator $U$ is given by

$$
U=\exp \left(i \frac{V\left(q_{n}\right) \epsilon}{\hbar}\right) \exp \left(i \frac{p_{n}^{2} \epsilon}{2 m \hbar}\right)
$$

Quantum mechanically, the energy $H^{0}\left(q_{n+1}, p_{n+1}\right)$ is not conserved, as expected since it was not conserved classically. It is remarkable however, that one can construct an "energy" (both at a quantum and classical level) that is conserved by the discrete evolution. This can be accomplished using the Baker-Campbell-Hausdorff formula

$$
\exp (X) \exp (Y)=\exp \left(X+Y+\frac{1}{2}[X, Y]+\frac{1}{12}([X,[X, Y]]+[Y,[Y, X]])+\cdots\right),
$$

and one can therefore write $U=\exp \left(\frac{i \epsilon}{\hbar} H_{\text {eff }}\left(q_{i}, p_{i}\right)\right)$ where $H_{\text {eff }}=H^{0}(q, p)+O\left(\epsilon^{2}\right)$, which is conserved under evolution. It is straightforward to write down a classical counterpart of this expression.

Let us now return to the classical theory and turn our attention towards systems with constraints in the continuum. In that case the discrete Lagrangian will be

$$
L(n, n+1)=p_{n}\left(q_{n+1}-q_{n}\right)-\epsilon H\left(q_{n}, p_{n}\right)-\lambda_{n B} \phi^{B}\left(q_{n}, p_{n}\right),
$$

where we assume we have $M$ constraints $B=1, \ldots, M$. 
We construct the appropriate canonically conjugate momenta using the first of (2.4)

$$
\begin{aligned}
& P_{n+1}^{q}=\frac{\partial L(n, n+1)}{\partial q_{n+1}}=p_{n}, \\
& P_{n+1}^{p}=\frac{\partial L(n, n+1)}{\partial p_{n+1}}=0 \\
& P_{n+1}^{\lambda_{B}}=\frac{\partial L(n, n+1)}{\partial \lambda_{(n+1) B}}=0 .
\end{aligned}
$$

Notice that the canonically conjugate momentum of the variable $q$ at instant $n+1$ is equal to the variable that superficially appeared as canonically conjugate at time $n$.

To determine the equations of motion for the system we start from the second set of equations $(2.4)$

$$
\begin{aligned}
& P_{n}^{q}=-\frac{\partial L(n, n+1)}{\partial q_{n}}=p_{n}+\epsilon \frac{\partial H\left(q_{n}, p_{n}\right)}{\partial q_{n}}+\lambda_{n B} \frac{\partial \phi^{B}\left(q_{n}, p_{n}\right)}{\partial q_{n}}, \\
& P_{n}^{p}=-\frac{\partial L(n, n+1)}{\partial p_{n}}=-\left(q_{n+1}-q_{n}\right)+\epsilon \frac{\partial H\left(q_{n}, p_{n}\right)}{\partial p_{n}}+\lambda_{n B} \frac{\partial \phi^{B}\left(q_{n}, p_{n}\right)}{\partial p_{n}}, \\
& P_{n}^{\lambda_{B}}=\phi^{B}\left(q_{n}, p_{n}\right) .
\end{aligned}
$$

Combining the last two sets of equations we get the equations of motion for the system

$$
\begin{aligned}
& p_{n}-p_{n-1}=-\epsilon \frac{\partial H\left(q_{n}, p_{n}\right)}{\partial q_{n}}-\lambda_{n B} \frac{\partial \phi^{B}\left(q_{n}, p_{n}\right)}{\partial q_{n}}, \\
& q_{n+1}-q_{n}=\epsilon \frac{\partial H\left(q_{n}, p_{n}\right)}{\partial p_{n}}+\lambda_{n B} \frac{\partial \phi^{B}\left(q_{n}, p_{n}\right)}{\partial p_{n}}, \\
& \phi^{B}\left(q_{n}, p_{n}\right)=0 .
\end{aligned}
$$

These equations appear very similar to the ones one would obtain by first working out the equations of motion in the continuum and then discretizing them. A significant difference, however, is that when one solves this set of equations the Lagrange multipliers get determined, they are not free anymore as they are in the continuum. We consider the constraint equation (2.12) and substitute $p_{n}$ by $(2.7)$

$$
\phi^{B}\left(q_{n}, P_{n+1}^{q}\right)=0 .
$$

We then solve (2.11) for $q_{n}$ and substitute it in the previous equation, one gets

$$
\phi^{B}\left(q_{n+1}, P_{n+1}^{q}, \lambda_{n B}\right)=0,
$$

and this constitutes a system of equations. Generically, these will determine

$$
\lambda_{n B}=\lambda_{n B}\left(q_{n+1}, P_{n+1}^{q}, v^{\alpha}\right),
$$

where the $v^{\alpha}$ are a set of free parameters that may arise if the system of equations is undetermined. The eventual presence of these parameters will signify that the resulting theory still has a genuine gauge freedom represented by freely specifiable Lagrange multipliers. We therefore see that generically when one discretizes constrained theories one gets a different structure than in the continuum in which Lagrange multipliers get determined.

The main advantage of this approach is that the resulting set of equations are consistent. The equations that in the continuum used to be constraints become upon discretization pseudoconstraints in that they relate variables at different instants of time and are solved by determining the Lagrange multipliers. The approach has been successfully applied in various example 
systems [35] including cosmologies [37] and used to evolve numerically the Gowdy space-time [38]. However, in pursuing those examples, particularly the Gowdy one which has a reasonable level of complexity, a drawback to this approach became quite evident, that led to a reformulation we will discuss next.

Before continuing it is worthwhile emphasizing some aspects of the discrete theories we have been constructing:

- The discrete Lagrangian is independent of the step $\varepsilon$.

- The discrete system is unconstrained, or at least has less constraints than the continuum one. If there are no constraints the evolution equations can be solved without ambiguities.

- Since the discrete system has more degrees of freedom than the continuum one there will exist trajectories that have no relation to those of the continuum (for instance trajectories far from the constraint surface).

- The Lagrange multiplier in the discrete theory is not free but becomes a well defined function of phase space. Its precise form will depend on the discretization, but in general it will vanish on the constraint surface. The closer the discrete evolution is to the constraint surface of the continuum theory, the smaller the values of the Lagrange multipliers.

- It could happen that the Lagrange multipliers in the discrete theory become complex.

Let us assume that the discrete theory is well defined. In that case we can proceed to quantize as we described. Supposing one can find the operator $\hat{U}$ that implements the evolution, one has a complete description of the quantum theory. The question now is: how do we take the continuum limit? The question is non-trivial since we don't have at hand the parameter $\varepsilon$ that controls the continuum limit. The classical continuum theory is achieved in the limit in which $q_{n+1} \rightarrow q_{n}$, which is equivalent to taking initial conditions close to the constraint surface. Quantum mechanically one would like something like " $\hat{q}_{n+1} \rightarrow \hat{q}_{n}$ ". Since the evolution operator is given (it does not depend on any parameter $\varepsilon$ ) such a condition can only be imposed at the level of the Hilbert space, considering states $|\psi\rangle$ such that $\hat{U}|\psi\rangle \rightarrow|\psi\rangle$. We will therefore say that the continuum theory is defined by states such that $\hat{U}|\psi\rangle=|\psi\rangle$. Later on, in the context of uniform discretizations, we will relate this condition with the definition of physical space in the Dirac quantization of constrained systems.

\subsection{Uniform discretizations}

The main drawback of the approach presented in the previous subsection is that the equations that determine the Lagrange multipliers are non-linear algebraic equations. Generically their solutions are therefore complex. Given that the Lagrange multipliers in general relativity are the lapse and shift, they cannot be complex. That means that the discrete theory can suddenly fail to approximate the continuous theory in a dramatic way as it becomes complex. Also, since the lapse and the shift determine the size of the temporal and spatial discrete steps, one typically would not want their values to become too large, otherwise the discrete equations will surely differ quite significantly or may even miss features from the continuum theory. Unfortunately one has no control on these matters. Given some initial data, the lapse and shift will evolve dynamically. If one encounters that they become complex, negative or too large one cannot do anything but change the initial data. This lack of control in the approximation clearly is a very undesirable feature, both for numerical evolutions but also for quantization.

To address the above problem we would like to make use of the considerable freedom that one has at the time of discretizing a theory. There exist infinitely many discrete theories that approximate a continuum one. We will use the freedom to demand that the evolution equations 
of the discrete theory take the form

$$
A_{n+1}=e^{\{\bullet, H\}}\left(A_{n}\right) \equiv A_{n}+\left\{A_{n}, H\right\}+\frac{1}{2}\left\{\left\{A_{n}, H\right\}, H\right\}+\cdots .
$$

The quantity $H$ is defined in the following way: Consider a smooth function of $N$ variables $f\left(x_{1}, \ldots, x_{N}\right)$ such that the following three conditions are satisfied: a) $f\left(x_{1}, \ldots, x_{N}\right)=0 \Longleftrightarrow$ $x_{i}=0 \forall i$ and otherwise $f>0$; b) $\frac{\partial f}{\partial x_{i}}(0, \ldots, 0)=0$; c) det $\frac{\partial^{2} f}{\partial x_{i} \partial x_{j}} \neq 0 \forall x$ and d) $f\left(\phi_{1}(q, p), \ldots\right.$, $\left.\phi_{N}(q, p)\right)$ is defined for all $(q, p)$ in the complete phase space. Given this we define $H(q, p) \equiv$ $f\left(\phi_{1}(q, p), \ldots, \phi_{N}(q, p)\right)$.

A particularly simple example is

$$
H(q, p)=\frac{1}{2} \sum_{i=1}^{N} \phi_{i}(q, p)^{2},
$$

essentially the "master constraint" of the "Phoenix project" [39]. The key observation is that the above evolution preserves the value of $H$. Therefore if one chooses initial data such that the constraints are small, they are guaranteed to remain small upon evolution. The evolution step is controlled by the value of $H$ and therefore by how small the constraints are. We call this approach uniform discretizations and the reader is referred to [40] for more details.

At first the chosen form for the evolution may sound counterintuitive. After all, if one gave data that satisfy the constraints exactly, there would be no evolution. Let us show that indeed the evolution approximates the continuum evolution well. Let $H$ as in the simple example above and take its initial value to be $H_{0}=\delta^{2} / 2$. We define $\lambda_{i}=\phi_{i} / \delta$, and therefore $\sum_{i=1}^{N} \lambda_{i}^{2}=1$. The evolution of the dynamical variable $q$ is given by

$$
q_{n+1}=q_{n}+\sum_{i=1}^{N}\left\{q_{n}, \phi_{i}\right\} \lambda_{i} \delta+O\left(\delta^{2}\right)
$$

and if we define $\dot{q} \equiv \lim _{\delta \rightarrow 0}\left(q_{n+1}-q_{n}\right) / \delta$, where we have identified the "time evolution" step with the initial data choice for $\delta$, one then has

$$
\dot{q}=\sum_{i=1}^{N}\left\{q, \phi_{i}\right\} \lambda_{i},
$$

and similarly for other dynamical variables. The particular values of the multipliers $\lambda_{i}$ depend on the initial values of the constraints $\phi_{i}$. Notice that as in the consistent discretizations approach, the Lagrange multipliers get determined, but they are well defined real functions of phase space. And note that the Lagrange multipliers are proportional to the constraints of the continuum theory. Therefore if the evolution is kept close to the constraint surface, the Lagrange multipliers will be small. If one chose initial data that exactly solved the continuum constraints, the Lagrange multipliers will vanish and the system does not evolve.

To illustrate that the above construction is actually feasible, let us consider a one-dimensional system, the parameterized harmonic oscillator. The continuum action for this system is

$$
S=\frac{1}{2} \int\left(\dot{x}^{2} / \dot{t}-x^{2} \dot{t}\right) d \tau,
$$

where we have chosen $m=k=1$ and the potential $V(x)=k x^{2} / 2$ as usual. The Lagrangian that yields the discrete evolution is given by

$$
L_{U}(x, t, X, T)=S(x, X, T-t)+\frac{1}{2}(T-t)^{2},
$$


where $S$ is Hamilton's principal function of the continuous system and we introduced the shorthand $t=t_{n}, T=t_{n+1}, x=x_{n}$ and $X=x_{n+1}$.

The equations for $X$ and $x$ give the equations of motion for a harmonic oscillator at a time $(T-t)$. When we work out the equations for $T$ and $t$ we have two terms

$$
P_{t}=p_{t}=\frac{\partial S(x, X, T-t)}{\partial T}+(T-t) .
$$

Since $S$ satisfies by the Hamilton-Jacobi equation, we have that

$$
\frac{\partial S(x, X, T-t)}{\partial T}=-H\left(x, p_{x}\right)=-\frac{1}{2}\left(p_{x}^{2}+x^{2}\right),
$$

so the equation for $T$ is

$$
T=t+p_{t}+H\left(x, p_{x}\right)=t+\phi(q, p) .
$$

That is, $\lambda_{d}(q, p)=\phi(q, p)$ as we expected. Note that this construction can be carried out for any parameterized system.

The constants of motion of the discrete theory are functions $O^{D}$ such that $\left\{O^{D}, H\right\}=0$. On the constraint surface, such functions coincide with the Dirac observables of the continuum theory, $O^{C}$. In order to see this, we note that

$$
\left\{O^{D}, H\right\}=\sum_{k} \phi_{k}\left\{O^{D}, \phi_{k}\right\}=0 .
$$

Let us take a point in phase space close to the constraint surface such that $\phi_{1}=\delta$ and $\phi_{k}=0$ $\forall k \neq 1$. From (2.14) we therefore see that $\left\{O^{D}, \phi_{1}\right\}=0$ at that point. Taking $\delta \rightarrow 0$ we conclude that $\left\{O^{D}, \phi_{1}\right\}=0$ for points on the constraint surface. Repeating this for the other constraints completes the proof. Conversely, to every Dirac observable of the continuum theory corresponds one or several constants of the motion of the discrete theory.

Let us conclude the classical part noting that in the case in which the constraints are Abelian it is possible to give an expression for the type 1 generating function of the canonical transformation that gives the discrete evolution. If the constraints $\phi_{k}$ are Abelian, then the discrete evolution be given by the Hamiltonian (2.13) can be understood as: "evolve for a time $\lambda_{1}$ with the field $X_{\phi_{1}}$; then evolve for a time $\lambda_{2}$ with the field $X_{\phi_{2}}$; etc." Here $\lambda_{i}(q, p)=\partial f /\left.\partial x_{i}\right|_{x_{k}=\phi_{k}(q, p)}$ is the Lagrange multiplier of the discrete theory. This can be done because the fields commute and because $\lambda_{i}(q, p)$ is constant throughout the evolution.

This construction corresponds to the evolution of the discrete Lagrangian

$$
L\left(q_{n}, q_{n+1}, \lambda_{1}, \ldots, \lambda_{r}\right)=S\left(q_{n}, q_{n+1}, \lambda_{1}, \ldots, \lambda_{r}\right)+g\left(\lambda_{1}, \ldots, \lambda_{r}\right),
$$

where $\lambda_{i}$ are the discrete Lagrange multipliers at the time $n, S$ is the Hamilton principal function of the system and $g$ satisfies that: 1) $g(0)=0$ and 2) the map $\lambda_{i} \rightarrow \frac{\partial g}{\partial \lambda_{i}}$ is the inverse of the map $x_{i} \rightarrow \frac{\partial f}{\partial x_{i}}$. In order to see this, note that the evolution equations of the $q$ 's yield the "exact" evolution with given Lagrange multipliers $\lambda_{k}$. The equations for the multipliers are

$$
0=\frac{\partial L}{\partial \lambda_{i}}=\frac{\partial S}{\partial \lambda_{i}}+\frac{\partial g}{\partial \lambda_{i}}
$$

From the Hamilton-Jacobi equation one has that $\partial S / \partial \lambda_{i}=-\phi_{i}$ and we recover the values of the multipliers that we had before.

Let us now discuss the quantum theory. Given how the system was put together, there is a natural candidate for the evolution operator

$$
\hat{U}=e^{-i \hat{H}} \text {. }
$$


The continuum limit is given by the quantum states $|\psi\rangle$ that satisfy

$$
\hat{U}|\psi\rangle=|\psi\rangle,
$$

which is equivalent to

$$
\hat{H}|\psi\rangle=0,
$$

which corresponds, at least classically, to the condition that the system satisfy the constraints. Condition (2.15) with $H$ given by (2.13) is the same condition used by Thiemann in his "master constraint" [39] proposal. The latter consists in finding the solutions of (2.15) as a means to finding the physical space of the theory. In that context the operator $\hat{H}$ is not interpreted as a Hamiltonian but as a "master constraint" that includes all the constraints of the problem. The idea is that states $|\psi\rangle$ that satisfy (2.15) will satisfy $\hat{\phi}_{k}|\psi\rangle=0 \forall k$.

The existence of solutions to (2.15) depends on if zero is in the spectrum of $H$. If it is not it will not be possible to find solutions and we will say that the quantum theory does not have a continuum limit. If zero is in the spectrum there will be a solution and the states that satisfy (2.15) are the physical space of states.

In order to define an inner product in the physical space of states one has to see if zero belongs in the discrete or continuous part of the spectrum of $H$. In the first case, the inner product is the same as in the kinematical Hilbert space. In the second, the states that satisfy (2.15) are not normalizable in the kinematical inner product and are generalized states. It is possible to introduce an inner product in this generalized space in the following way. One takes the projector $\hat{P}$ from the kinematical to the physical Hilbert space. The internal product between two states $\left|\psi_{1}\right\rangle_{\mathrm{Ph}}$ and $\left|\psi_{2}\right\rangle_{\mathrm{Ph}}$ of the physical Hilbert space such that $\left|\psi_{i}\right\rangle_{\mathrm{Ph}}=\hat{P}\left|\psi_{i}\right\rangle$ with $\left|\psi_{i}\right\rangle$ states of the kinematical Hilbert space is given by

$$
\mathrm{Ph}\left\langle\psi_{1} \mid \psi_{2}\right\rangle_{\mathrm{Ph}}=\left\langle\psi_{1}|\hat{P}| \psi_{2}\right\rangle .
$$

Such a projector can be constructed from the evolution operator in the following manner

$$
\hat{P} \equiv \lim _{n \rightarrow \infty} C_{n} \hat{U}^{n}
$$

with appropriate constants (c-numbers) $C_{n}$ such that $\lim _{n \rightarrow \infty}\left(C_{n+1} / C_{n}\right)=1$. One then has that $\hat{U} \hat{P}=\hat{P}$ and therefore $\hat{U} \hat{P}|\psi\rangle=\hat{P}|\psi\rangle$ for all states $\mid \psi>$ of the kinematic Hilbert space.

The quantization of the resulting discrete theories have important conceptual simplifications with respect to the original continuum theory. First of all, the theories are free of constraints. The latter are approximately enforced by the evolution and can be made as small as one wishes by choosing initial data. But one does not need to be concerned with issues like constraint algebras anymore. One will be quantizing a classical theory that, although it approximates the theory of interest with arbitrary precision, is conceptually very different.

To quantize these theories we start by picking a kinematical Hilbert space that will be given by square integrable functions of the configuration variables $\Psi(q)$. On this space one defines operators $\hat{Q}$ and $\hat{P}$ as usual. To construct the operators at other time levels one introduces a unitary operator $\hat{U}$ that we will define later, with the following properties

$$
\hat{Q}_{n} \equiv \hat{U}^{-1} \hat{Q}_{n-1} \hat{U}=\hat{U}^{-n} \hat{Q}_{0} \hat{U}^{n}, \quad \hat{P}_{n} \equiv \hat{U}^{-1} \hat{P}_{n-1} \hat{U}=\hat{U}^{-n} \hat{P}_{0} \hat{U}^{n} .
$$

The evolution operator is given by $\hat{U}=e^{-i \hat{H} / \hbar}$. The operator $\hat{U}$ may also be determined by requiring that the fundamental operators satisfy an operatorial version of the evolution equations

$$
\hat{Q}_{n} \hat{U}-\hat{U} Q_{n+1}\left(\hat{Q}_{n}, \hat{P}_{n}\right)=0, \quad \hat{P}_{n} \hat{U}-\hat{U} P_{n+1}\left(\hat{Q}_{n}, \hat{P}_{n}\right)=0,
$$

and this provides a consistency condition that aids in the construction of $\hat{U}$. 
We recall that classically $H=0$ if and only if the constraints $\phi_{i}=0$. One can define naturally the physical space of the continuum theory in a way that does not require that we refer to the constraints directly. Since we know that $\hat{U}=\exp (-i \hat{H} / \hbar)$, a necessary condition satisfied by the states of the physical space of the continuum theory, $\psi \in \mathcal{H}_{\text {phys }}$ is given by $\hat{U} \psi=\psi$. More precisely the states $\psi$ of $\mathcal{H}_{\text {phys }}$ should belong to the dual of a space $\Phi$ of functions sufficiently regular on $\mathcal{H}_{k}$. That is, the states $\psi \in \mathcal{H}_{\text {phys }}$ satisfy

$$
\int \psi^{*} \hat{U}^{\dagger} \varphi d q=\int \psi^{*} \varphi d q
$$

where $\varphi \in \Phi$. This condition characterizes the quantum physical space of a constrained continuum theory without needing to implement the constraints as quantum operators. This is an important advantage in situations where implementing the constraints as quantum operators could be problematic, as for instance when they do not form a Lie algebra. This is the case in general relativity where the constraint algebra has structure functions. For more details on the quantization see [40].

\subsubsection{Examples}

We discuss now two significant examples that will illustrate the main two possibilities when implementing the construction outlined here. In the first example, one finds that the quantum master constraint includes the zero eigenvalue in its kernel. There the technique reproduces the results of the ordinary Dirac quantization technique. In the second example the zero eigenvalue is not in the kernel. The resulting quantum theory has a fundamental level of discreteness and will only recover semiclassically the continuum theory in certain circumstances. In this example the Dirac quantization is also problematic. We believe this example illustrates the most likely situation one will face in gravity: the resulting quantum theory will have a fundamental level of discreteness and classical general relativity will only be recovered in long length scales compared to the Planck length. This is a point of view that many people have advocated for an ultimate theory of quantum gravity over the years.

The two examples are taken from [40].

\subsubsection{Model with continuum limit}

Here we will consider a model constrained system with non-Abelian constraints that form a Lie algebra. We consider a mechanical system with configuration manifold $R^{3}$, coordinatized by $q^{i}$, $i=1,2,3$ and 3 non-commuting "angular momentum" constraints

$$
C^{i}=L^{i} \equiv \epsilon^{i j k} q^{j} p^{k}, \quad i=1,2,3,
$$

where the $p^{i}$ 's are the momenta conjugate to the $q^{i}$ 's and we assume Einstein's summation convention on repeated indices. The three constraints are not independent. Vanishing angular momentum is equivalent to requiring $q^{i}=\lambda p^{i}$ with $\lambda$ arbitrary, which implies two conditions to be satisfied by the phase space variables. The constraint surface is four dimensional and therefore the system has two independent observables. We can choose to construct the Hamiltonian starting from two independent constraints or one could choose to use a more symmetric, yet redundant, form of the constraints, which will be our choice. The Hamiltonian that will dictate evolution is

$$
H=\sum_{i=1}^{3} \frac{\left(L^{i}\right)^{2}}{2 k},
$$


where $k$ is a constant with units of action. The resulting discrete evolution equations are

$$
\begin{aligned}
& q_{n+1}^{i}=q_{n}^{i}+\epsilon_{i j k} \frac{q^{k} L^{j}}{k}+\epsilon_{i j k} \epsilon_{j m n} q^{m} \frac{L^{n} L^{k}}{k^{2}}+\cdots, \\
& p_{n+1}^{i}=p_{n}^{i}+\epsilon_{i j k} \frac{p^{k} L^{j}}{k}+\epsilon_{i j k} \epsilon_{j m n} p^{m} \frac{L^{n} L^{k}}{k^{2}}+\cdots
\end{aligned}
$$

One obtains the continuum limit by setting $H=\delta^{2} / 2$ and defining $\lambda^{i}=L^{i} /(\delta \sqrt{k})$. Taking the limit as explained before, one gets

$$
\dot{q}^{i}=\epsilon_{i j k} \frac{q^{k} \lambda^{j}}{\sqrt{k}}, \quad \dot{p}^{i}=\epsilon_{i j k} \frac{p^{k} \lambda^{j}}{\sqrt{k}} .
$$

The components $L^{i}$ are constants of the motion and therefore $\lambda^{i}$ are constant. There exist three constants more: $q \cdot q=q^{i} q^{i}, p \cdot p=p^{i} p^{i}, q \cdot p=q^{i} p^{i}$. These are not independent since $L^{2}-\left((q \cdot q)(p \cdot p)-(q \cdot p)^{2}\right)=0$. One therefore has five independent constants of the motion of the discrete theory. In the continuum limit the $L^{i}$ 's vanish and one has two independent constants of motion, for instance $q \cdot q$ and $q \cdot p$. In the continuum, the trajectories are arbitrary trajectories on a sphere. In the discrete theory, when one takes the continuum limit one obtains trajectories that correspond to arbitrary circumferences on the sphere, since the $\lambda^{i}$ 's are constant. The constraint surface is therefore completely covered, but not all orbits of the continuum theory are recovered. This however, is not problematic since we can recover all physical information with the trajectories obtained.

To quantize the system, we will start with an auxiliary Hilbert space $L_{2}\left(R^{N}\right)$ on which the operators $\hat{q}^{i}$ act as multiplication operators and the momenta as derivatives

$$
\hat{q}^{i} \psi(q)=q^{i} \psi(q), \quad \hat{p}^{i} \psi(q)=-i \hbar \partial_{i} \psi(q),
$$

and immediately construct the unitary operator

$$
\hat{U}=\exp \left(-i \frac{\hat{L}^{2}}{2 k \hbar}\right),
$$

which recovers (up to terms of order $\hbar$ ) the classical discrete evolution equations as operatorial relations so the correspondence principle is satisfied.

To compute the projector we use the formula (2.16) and, work with a basis labeled by the radial and angular momentum eigenvalues. The projector is given by

$$
\hat{P}|n, \ell, m\rangle=\delta_{\ell 0}|n, \ell, m\rangle=\delta_{\ell 0}|n, 0,0\rangle,
$$

which can be rewritten as,

$$
\hat{P}=\sum_{n=0}^{\infty}|n, 0,0\rangle\langle 0,0, n| .
$$

The continuum limit is therefore immediately achieved. The physical space is the space of vanishing angular momentum and on it the constraints of the continuum theory are automatically satisfied. We have therefore recovered the usual Dirac quantization. The physical space is given by the square integrable functions depending on the radial variable $|q|$. The physical inner product is therefore induced by the kinematical inner product on the physical space of states. 


\subsubsection{A model with non-compact gauge group}

We will now consider a system with non-Abelian constraints that form a Lie algebra that is noncompact, associated with the $S O(2,1)$ group. It has the same phase space as in the previous example, except that the metric will be given by $\operatorname{diag}(-1,1,1)$. The constraints are

$$
C_{i}=L_{i} \equiv \epsilon_{i j}{ }^{k} q^{j} p_{k}, \quad i=0,1,2 .
$$

One has to be careful about upper and lower indices since the metric is non-trivial. Just like before, the three constraints are not independent and the constraint surface is four dimensional and the system has two independent observables. The canonical evolution is given through the master constraint

$$
H=\frac{L_{i} L^{i}+2 L_{0}^{2}}{2 k}
$$

where $k$ is a constant with units of action. The discrete evolution equations are

$$
\begin{aligned}
& q_{n+1}^{i}=q_{n}^{i}+\epsilon^{i}{ }_{j k} \frac{q^{k} L^{j}}{k}-2 \epsilon_{0 k}^{i} \frac{q^{k} L^{0}}{k}+\cdots, \\
& p_{n+1}^{i}=p_{n}^{i}+\epsilon^{i}{ }_{j k} \frac{p^{k} L^{j}}{k}-2 \epsilon_{0 k}^{i} \frac{p^{k} L^{0}}{k}+\cdots .
\end{aligned}
$$

The continuum limit is achieved setting $H=\delta^{2} / 2$. Defining $\lambda^{i}=L^{i} /(\delta \sqrt{k})$ for $i=1,2$ and $\lambda^{0}=-L^{0} /(\delta \sqrt{k})$ we have, taking the limit as explained before

$$
\dot{q}^{i}=\epsilon_{j k}^{i} \frac{q^{k} \lambda^{j}}{\sqrt{k}}, \quad \dot{p}^{i}=\epsilon_{j k}^{i} \frac{p^{k} \lambda^{j}}{\sqrt{k}} .
$$

We note that although $L_{0}$ is a constant of the motion, the other components are not. However $L_{1}^{2}+L_{2}^{2}$ is a constant, so $L_{1}$ and $L_{2}$ rotate around $L_{0}$ throughout the evolution. There exist three further constants $q \cdot q=q^{i} q_{i}, p \cdot p=p^{i} p_{i} q \cdot p=q^{i} p_{i}$. These are not independent since $L \cdot L-\left((q \cdot q)(p \cdot p)-(q \cdot p)^{2}\right)=0$. One therefore has four independent constants of the motion of the discrete theory. In the continuum limit two vanish and one is left with two constants of motion, for instance $q \cdot q$ and $q \cdot p$. The trajectories in the continuum are arbitrary trajectories on two hyperboloids, one space-like and one time-like. The continuum limit of the discrete theory yields trajectories that correspond to particular choices of the Lagrange multipliers, depending on the initial conditions chosen.

The quantization of this model (and similar ones) is known to have subtleties [39, 41, 42]. The central problem is that if one promotes the constraints to operators on the usual Hilbert space, they do not have a vanishing eigenvalue in their spectra. This can happen, but usually the resolution is to extend the Hilbert space by including an improper basis of eigenvectors. This can be done in this case, but one finds that the spectrum again does not contain zero. More specifically, the continuum spectrum has eigenvalues larger than $\hbar^{2} / 4$. One can find eigenvectors with eigenvalues smaller than $\hbar^{2} / 4$, but they do not arise as limit of functions of the Hilbert space, i.e. they cannot form part of an improper basis of the Hilbert space. One can adopt the point of view that nevertheless the eigenvectors with zero eigenvalue are the "physical space" of the theory, essentially abandoning the idea that the physical space arises as a suitable limit of the kinematical space. This was suggested in [41, 42]. From the point of view of our approach this does not suffice, since we wish to construct the physical space of states starting from the quantum kinematical space, taking a limit. As we mentioned before, lacking the zero eigenvalue in the spectrum of the Hamiltonian yields our prescription for the projector on the physical space useless. With this in mind, the most satisfactory solution is 
the one chosen in [39], where one chooses as physical space the eigenvectors that have $\hbar^{2} / 4$ as eigenvalue. Another attractive possibility in the discrete approach is not to take the continuum limit and retain a level of fundamental discreteness. This is very natural in a model where it is difficult to achieve a vanishing eigenvalue for the constraints, a natural minimum existing for their eigenvalues. As we argued before, such models can approximate the semiclassical physics of the theory of interest with some restrictions on the type of states considered. There can therefore be viewed as the best thing one can do in terms of having an underlying quantum theory for the model that approximates the classical physics of interest.

\subsubsection{Other examples}

The above two examples capture the essence of the technique in which we see in one case that we recover the traditional Dirac quantization, whereas in the other case, where even the Dirac procedure faces issues, we obtain a satisfactory solution. The technique has also been applied in $2+1$ gravity [40] where one recovers the traditional quantization and more recently to the study of spherically symmetric gravity coupled to a spherically symmetric scalar field [43]. This is a challenging example with a rich and complex dynamical structure and infinite degrees of freedom. Up to now only low energy regimes close to flat space have been studied . One cannot achieve a continuum limit and one is left with a theory with a fundamental level of discreteness that nevertheless approaches semiclassically general relativity very well, as we had anticipated.

\section{$3 \quad$ Improved and perfect actions}

In this section we review the mechanism of symmetry breaking in the covariant language, i.e. within Regge Calculus. We also describe the perfect action approach in order to cope with the arising problems, and demonstrate the connections to the renormalization group.

\subsection{Diffeo breaking in Regge}

We consider the discretization of General Relativity via Regge's method, and demonstrate in which sense the diffeomorphisms of the continuum are broken. In Regge calculus $d$-dimensional space-time is discretized by a triangulation $\mathcal{T}$ consisting of simplices $\sigma$, and the metric information is encoded as edge lengths ${ }^{3} l_{e}$ of the simplices $\sigma$. By specifying the edge lengths the geometry of the simplex is uniquely determined, and the discrete analogue of the EinsteinHilbert action (1.1), called the Regge action, is given by

$$
S_{\mathrm{R}}\left[l_{e}\right]=\sum_{h \in \mathcal{T}^{\circ}} V_{h} \underbrace{\left(2 \pi-\sum_{\sigma \supset h} \theta_{h}^{\sigma}\right)}_{=: \epsilon_{h}}+\sum_{h \in \partial \mathcal{T}} V_{h} \underbrace{\left(\pi-\sum_{\sigma \supset h} \theta_{h}^{\sigma}\right)}_{=: \psi_{h}}-\Lambda \sum_{\sigma} V_{\sigma},
$$

where the first sum ranged over all $d-2$-simplices (called hinges) in the interior, and the second sum ranges over all hinges in the boundary of $\mathcal{T}$. The curvature is encoded in the excess of the sum of dihedral angles $\theta_{h}^{\sigma}$ around a hinge $h$ with respect to $2 \pi$ (see Fig. 1), and denoted as deficit angle $\epsilon_{h}$. Similarly, for a hinge $h \in \partial \mathcal{T}$ on the boundary, $\psi_{h}$ encodes the extrinsic curvature of the boundary surface $\partial \mathcal{T}$ in $\mathcal{T}$. Also, $V_{h}$ denotes the $(d-2)$-dimensional volume of the hinge $h$, and $V_{\sigma}$ the $d$-dimensional volume of the simplex $\sigma$.

\footnotetext{
${ }^{3}$ For simplicity we consider only Riemannian metrics in what follows, although Lorientzian metrics can equally well be treated by considering the edge length squares $l_{e}^{2}$, which can e.g. be negative if the edge is time-like. Special care has to be taken when considering the causal structure of the triangulation [44].
} 

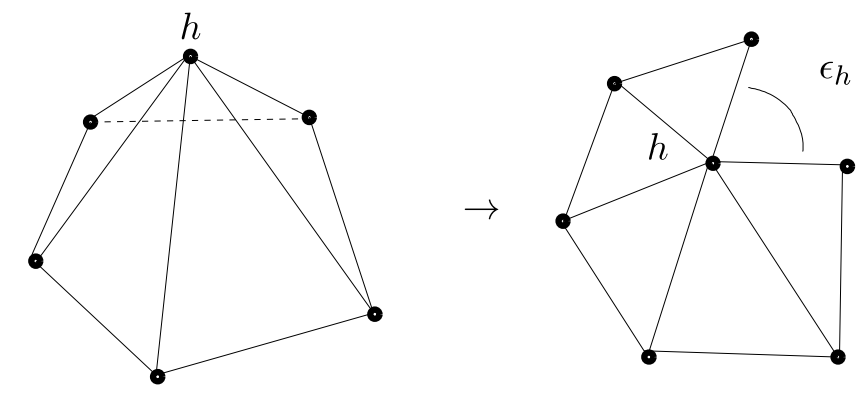

Figure 1. Deficit angle $\epsilon_{h}$ situated at a $(d-2)$-dimensional simplex (hinge) $h$.

The boundary terms and the Schlaefli-identity [45] ensure that the equations of motion are given by

$$
\frac{\partial S_{\mathrm{R}}}{\partial l_{e}}=\sum_{h \supset e} \frac{\partial V_{h}}{\partial l_{e}} \epsilon_{h}-\Lambda \sum_{\sigma \supset e} \frac{\partial V_{\sigma}}{\partial l_{e}}=0
$$

for all interior edges $e \in \mathcal{T}^{\circ}$, which are conditions for the interior edge lengths $l_{e}$ with fixed boundary edge lengths.

In the continuum theory, i.e. GR, the Einstein equations do not determine uniquely a solution for the interior metric, but only up to diffeomorphism. Also, the Einstein equations contain as subset consistency conditions for the boundary metric, which do not contain dynamical information, but constitute constraints. In the discrete setting the same situation appears in the special case of $d=3$ and $\Lambda=0$ : The hinges $h$ and the edges $e$ coincide, and therefore the Regge equations (3.2) simply become

$$
\epsilon_{e}=0
$$

For given boundary lengths $l_{e}$, the equations (3.3) have in general infinitely many solutions for the interior lengths, since there are infinitely many ways of filling up a portion of flat 3 -dimensional space with tetrahedra. Of a given solution for the $l_{e}$, another solution can be constructed by vertex translation, and it can be shown that this transformation between solutions is generated by the discrete Bianchi-identities [24, 25, 46], which therefore corresponds exactly to a diffeomorphism in this case. Also, as part of the Regge equations, the boundary lengths have to satisfy a constraint, which demands that the $2 d$ boundary triangulation can be immersed in flat $3 d$ space [28], which is again completely similar to the continuum case.

However, as soon as one leaves the case of $d=3$ or $\Lambda=0$, the situation changes. This can be seen as follows: The non-uniqueness of the solutions to (3.3) manifest themselves in zero Eigenvalues of the Hessian matrix $H_{e e^{\prime}}=\partial^{2} S_{\mathrm{R}} / \partial l_{e} \partial l_{e^{\prime}}$. These zeros are precisely what makes the Legendre transformation singular, indicating the presence of gauge symmetries [29]. The same zeros appear in the Hessian for $4 d, \Lambda=0$, whenever evaluated on a flat solution $\epsilon_{t}=0$ (where $t$ denotes the triangles of $\mathcal{T}$ ), and the zero modes correspond to the diffeomorphism gauge modes of linearized gravity [27].

It was well-known for some time that for $4 d$ and $\Lambda=0$ the vertex displacement symmetry, around the flat solution, is satisfied at least up to terms of order $\epsilon^{2}$ [26, 27]. In [29] this bound was made sharp by showing that the Hessian is non-degenerate, i.e. has no zero Eigenvalues, in the case of $3 d, \Lambda \neq 0$, and in $4 d, \Lambda=0$ whenever the boundary data is such that the solution has internal curvature. In fact, the smallest Eigenvalue scales with $\epsilon^{2} \sim \Lambda^{2}$ in the case of $3 d$, as well was with $\epsilon^{2}$ (where $\epsilon$ is a deficit angle at an internal edge) in $4 d$ (see Fig. 2). As soon as the solution to (3.2) exhibits curvature, there is no continuous symmetry relating different solutions anymore, and although in some cases there are different discrete solutions [47], these 


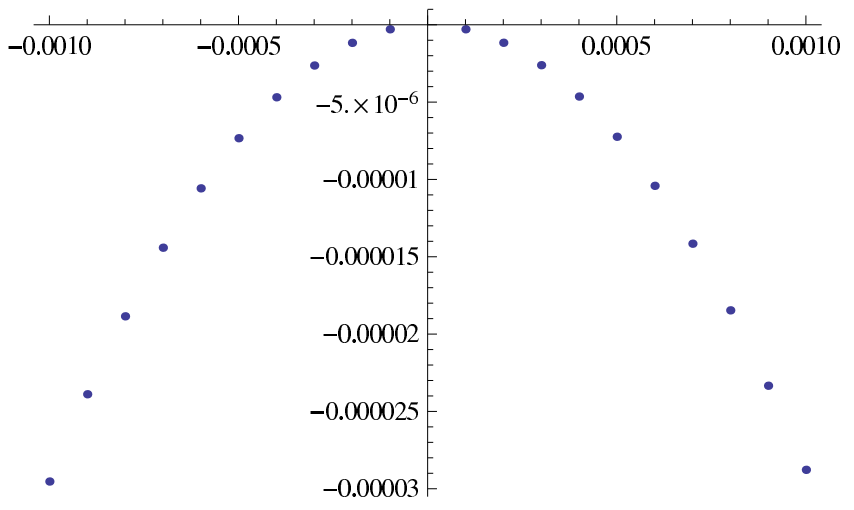

Figure 2. The lowest eigenvalue of the Hessian as a function of deviation from flatness (proportional to $\epsilon$ ), in a particular $4 d$-triangulation with one inner vertex, discussed in [29].

are rather a discretization artifact. In most cases the solution for given boundary data is unique. Moreover, the part of the Regge equations which, in the flat case, constituted the constraints, i.e. which involved only the initial data on one time step $^{4}$, acquired a weak coupling between data on subsequent time slices, i.e. turned from a constraint into a (weakly) dynamical equation. These equations are therefore called pseudo-constraints.

The uniqueness of solutions in cases with nonvanishing curvature (i.e. the breaking of the continuum gauge symmetry) leads to a vast overcounting of degrees of freedom. Consider e.g. the case of $3 d$ Regge with $\Lambda \neq 0$. For a fixed triangulation, since there are no constraint equations for the boundary data, not only can one prescribe many more initial conditions, each of which lead to a unique solution of motion, since there are infinitely many different triangulations (even with the same boundary triangulation), each of which produce physically different solutions, the discrete theory has many more than just the finitely many (topological) degrees of freedom that the continuum theory has.

The breaking of symmetries in the discrete therefore leads to the emergence of pseudo-degrees of freedom, leading to several problems: The first is an interpretational one, which has also been realized in other discrete, in particular numerical, approaches to GR [52]: It is unclear how to distinguish the pseudo degrees of freedom from the actual physical ones. It is therefore difficult to extract the physical content from a numerical solution, e.g. disentangling actual oscillations of gravitational waves from oscillations of the chosen coordinate system.

Another problem arises within any attempt to build a quantum gravity theory based on Regge discretizations: Generically, breaking of gauge symmetries within the path integral measure leads to anomalies, in particular problematic in interacting theories [53]. In quantum theories based on Regge calculus, the path integral will, for a very fine triangulation, contain contributions of lots of almost gauge equivalent discrete metrics, each with nearly the same amplitude. Hence the amplitude will not only contain infinities of the usual field theoretic nature, but also coming from the effective integration over the diffeomorphism gauge orbit. This is in particular a problem for the vertex expansion of the spin foam path integral, as advocated in [54]. The triangulations with many vertices will contribute much more to the sum than the triangulations with few vertices, so that, at every order, the correction terms dominate, rendering the whole sum severely divergent. We will see this phenomenon occur in Section 3.4, in the case of a toy model.

In the following we describe an attempt to circumvent the above problems, by constructing a discrete action with the correct amount of symmetries. The main technical tool for this is coarse graining.

\footnotetext{
${ }^{4}$ Here the different time steps were taken as a series of so-called tent moves [48]. For a canonical framework for simplicial theories which builds on Pachner moves, see also [32, 49, 50, 51].
} 


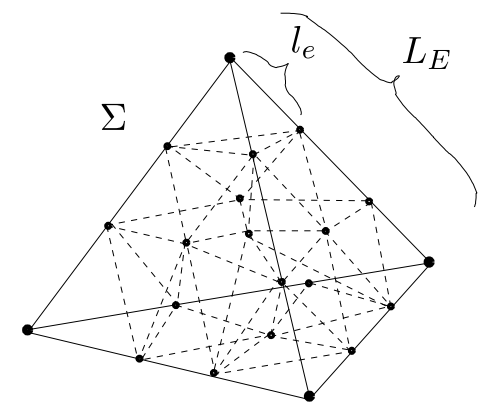

Figure 3. A tetrahedron $\Sigma$ subdivided into smaller tetrahedra $\sigma$. The length $L_{E}$ of a coarse edge $E$ is the sum of all the lengths $l_{e}$ of edges $e \subset E$ that constitute $E$.

\subsection{The coarse graining idea: classical}

A toy model for the breaking of diffeomorphism symmetry in Regge calculus, which has been investigated in $[23,33,55]$ is parameterized mechanics, in which time $t$ itself is treated as a configuration variable, which, together with $q$, evolves with respect to an auxiliary parameter $s$. The broken symmetry in the discrete case is invariance under reparametrization $s \rightarrow s^{\prime}(s)$, which is effectively diffeomorphism symmetry in $1 d$. In this case, there is a way of constructing an action for the discrete theory (i.e. depending on $\left(t_{n}, q_{n}\right)$ ), which nevertheless exhibits the exact symmetry from the continuum theory. Given a discretization of the continuum action $S^{(0)}$, this perfect action can be constructed iteratively by a coarse graining procedure, resulting in a sequence of discrete actions $S^{(n)}$ converging to the perfect action $S^{(\infty)}$ in the limit.

A similar construction can be employed in principle within the context of Regge calculus, and we show how this procedure, described in [23], is able to cure the problems described above in the case of $3 d$ Regge with $\Lambda \neq 0$. Consider a $3 d$ triangulation $\mathcal{T}$ (capturing the macroscopic degrees of freedom) and a refined triangulation $\tau$, such that each edge $E \in \mathcal{T}$ can be composed of edges $e \in \tau$ (see Fig. 3), capturing the microscopic degrees of freedom. The effective (or improved) action $S_{\mathcal{T}, \tau}\left[L_{E}\right]$, which lives on $\mathcal{T}$, is constructed by putting the Regge action on $\tau$ and integrating out the microscopic degrees of freedom. To this behalf, one solves the Regge equations for the $l_{e}, e \in \tau$, with fixed edge lengths

$$
\sum_{e \subset E} l_{e} \stackrel{!}{=} L_{E}
$$

One therefore adds the conditions (3.4) to the Regge action (3.1) via Lagrange multipliers $\alpha_{E}$, extremizing

$$
S\left[l_{e}\right]=\sum_{e} l_{e} \epsilon_{e}-\Lambda \sum_{\sigma \in \tau} V_{\sigma}+\sum_{E} \alpha_{E}\left(L_{E}-\sum_{e \subset E} l_{e}\right) .
$$

Note that the action (3.5) depends on the $L_{E}$ as parameters. The effective action $S_{\mathcal{T}, \mathcal{T}^{\prime}}\left[L_{E}\right]$ is then defined as the value of the action (3.5) evaluated on a solution, i.e.

$$
S_{\mathcal{T}, \tau}\left[L_{E}\right]:=S\left[l_{e}\right]_{\mid \frac{\partial S}{\partial l_{e}}=\frac{\partial S}{\partial \alpha_{E}}=0} .
$$

One can show straightforwardly that

$$
S_{\mathcal{T}, \tau}\left[L_{E}\right]=\sum_{E} L_{E} \alpha_{E}+2 \Lambda \sum_{\Sigma \in \mathcal{T}} V_{\Sigma}
$$


with $V_{\Sigma}:=\sum_{\sigma \subset \Sigma} V_{\sigma}$ for a simplex $\Sigma \in \mathcal{T}$. Note that both $\alpha_{E}$ and $V_{\Sigma}$ are a priori complicated functions of the $L_{E}$, which have to be determined by the equations of motion for $S$. Without knowing their form explicitly, setting

$$
\Theta_{E}^{\Sigma}:=\sum_{\sigma \supset e, \sigma \subset \Sigma}\left(\theta_{e}^{\sigma}-\Lambda \frac{\partial V_{\sigma}}{\partial l_{e}}\right),
$$

which does not depend on the choice of $e \subset E$ by the equations of motion, one can derive the identities

$$
\begin{aligned}
& \alpha_{E}=2 \pi-\sum_{\Sigma \supset E} \Theta_{E}^{\Sigma}, \\
& \sum_{E \subset \Sigma} L_{E} \frac{\partial \Theta_{E}^{\Sigma}}{\partial L_{E^{\prime}}}=2 \Lambda \frac{\partial V_{\Sigma}}{\partial L_{E^{\prime}}} .
\end{aligned}
$$

Note that (3.7) implies that the equations of motion for the $L_{E}$ as given by the effective action $S_{\mathcal{T}, \tau}$ are simply

$$
\frac{\partial S_{\mathcal{T}, \tau}}{\partial L_{E}}=\alpha_{E}=0
$$

Not only is (3.7) precisely of the form of the Schlaefli identity for simplices of constant curvature $\Lambda$ [45], but one can also show [23] that in the limit of $\tau$ becoming very fine with respect to $\mathcal{T}$ in a controlled way, the functions $\Theta_{E}^{\Sigma}, V_{\Sigma}$ converge to the dihedral angle and the volume within a tetrahedron of constant curvature $\Lambda$, with edge lengths $L_{E}$. So the perfect action, as the limit of infinite refinement $\tau \rightarrow \infty$, is given by the Regge action with simplices of constant curvature $\Lambda$

$$
S_{\mathcal{T}, \infty}=\sum_{E} L_{E} \epsilon_{E}+2 \Lambda \sum_{\Sigma} V_{\Sigma}
$$

Indeed, the equations of motion for (3.8) are simply $\epsilon_{E}=0$, so the solutions describe a metric of constant curvature everywhere. Just as in the case for $\Lambda=0$, where $\epsilon_{E}=0$ allows for arbitrary subdivision of flat space into flat simplices, resulting in the vertex displacement symmetry, in the $\Lambda \neq 0$ case $\epsilon_{E}=0$ allow for arbitrary subdivision of constantly curved space into constantly curved simplices, and the same vertex displacement symmetry appears. Hence, unlike the Regge action (3.1), the perfect action (3.8) exhibits the correct symmetries of the continuum theory ${ }^{5}$.

The same coarse graining procedure works for the $\Lambda \neq 0$ sector in $4 d$, which is such that the solution to the equations of motion results in constant curvature [23]: also in this case the Regge action (3.1) converges to its analogue with constantly curved 4-simplices by means of the procedure described above.

\subsection{Feasibility}

The procedure for constructing the perfect action for a discrete system requires the solution of the equations of motion for a very fine discretization. Even for the case for $3 d, \Lambda \neq 0$ described above there is no analytic formula for the solutions. The simplicity of the system, and in particular the structural equation (3.7) allowed to prove that the sequence of effective actions $S_{\mathcal{T}, \tau}$ converges to (3.8) in the limit of infinitely fine $\tau$. For more complicated systems (in particular for discrete GR in $4 d$ ), it might not be feasible to try and compute the perfect action

\footnotetext{
${ }^{5}$ In fact, (3.1) for $3 d$ consists of the first two terms in the $\Lambda$-expansion of (3.8), i.e. up to $O\left(\Lambda^{2}\right)$.
} 
explicitly. In particular, in the known cases the perfect actions are always closely related to the Hamilton's principal function of the continuous system [23], the explicit knowledge of which is equivalent to having control over the solution space of the continuum theory, which is definitely not the case for $4 d$ GR.

Still, the concept of a perfect action is a very powerful tool in numerical studies of lattice QCD [56, 57], where it is used to minimize discretization artifacts. Here, the perfect action is not known explicitly, but there exist very good numerical and analytical approximations to it. In [58], a procedure was described for how to compute the perfect action approximately within a vicinity of a point in configuration space. Here it was found that, if one concentrates on the perfect action around a solution $\left\{l_{e}^{(0)}\right\}$ which exhibits the exact symmetry of the continuum (as e.g. the case for flat space in $4 d$ Regge for $\Lambda=0$ ), the gauge symmetries are satisfied to linear order in the perturbative expansion. However, the breaking of the gauge symmetries to higher order imposes consistency conditions on the actual state around which one perturbs. Within the gauge equivalence class $\left\{l_{e}^{(0)}\right\}$ of solutions which all solve the equations of motion, there is only one particular solution around which the perturbative expansion is consistent. In fact, this solution is singled out by the condition that the Hamilton's principal function to second order depends minimally on the gauge parameters [32].

Computing the perfect action to lowest non-trivial order for linear systems has been performed in [58], where it could be shown that, for linearized gravity the perfect action (to linear order) is invariant under coarse graining, and displays the correct amount of symmetries in $3 d$. In $4 d$, there appear non-localities, which make the results more complicated, and which could in principle be dealt with by Migdal-Kadanoff-type approximation schemes [59, 60].

\subsection{Coarse graining: quantum}

The broken diffeomorphism symmetry of discretized theories can lead to interpretational problems in the classical realm, as we have argued in the last section. Here one cannot easily distinguish the physical degrees of freedom from the ones that arise by breaking the gauge symmetry. The finer the discretization, the better the approximate symmetries (i.e. the closer the minimal Eigenvalues in the Hessian are to actually vanish), and the closer the pseudoconstraints are to the actual constraints of the continuum theory.

Within the corresponding quantum theory, the problem becomes even more severe. For finite discretization, the additional degrees of freedom, which become gauge in the continuum limit, are being treated as physical and are summed over in the path integral, which lead to unphysical singularities ${ }^{6}$.

Consider for instance the example of parametrized $1 d$ mechanics, with continuum variables $q(s)$ and $t(s), s$ being an auxiliary gauge parameter. Due to the gauge symmetry (i.e. reparametrization-invariance) only the dependence $q(t)$ is physical, rather than $q$ and $t$ itself, and one indeed gets the correct answer for the propagator if one drops the integration over $t$ within the path integral, and only integrates over $q$.

The discrete configuration variables are $\left(t_{n}, q_{n}\right)$ and $n=1, \ldots, N$. In the path integral

$$
K^{(0)}\left(t_{0}, q_{0}, t_{n}, q_{n}\right)=\int_{\mathbb{R}^{2 N}} d t_{n} d q_{n} e^{\frac{i}{\hbar} S_{\text {discrete }}\left(t_{n}, q_{n}\right)}
$$

the summation over both $t_{n}$ and $q_{n}$ have to be performed, since there is no gauge symmetry between the variables, if one uses a generic discretized approximation to the continuum action. In the limit of finer and finer discretization (i.e. letting $N$ go to infinity), the contribution coming from the integration over the $t_{n}$ approaches the integral over the gauge group (which

\footnotetext{
${ }^{6}$ As opposed to e.g. UV or IR divergencies in QFT, which carry information about the physical properties of the system.
} 
is infinite ${ }^{7}$ ). While in this particular case the problem can be solved by just neglecting the $t_{n}$-integration by hand, this relies on an apriori knowledge of which variables remain physical and which become gauge within the continuum, i.e. a separation of the configuration variables into $t$ and $q$. For more complicated systems (in particular within GR), this separation is not possible.

However, given the propagator $K^{(0)}$ as by (3.9), it is possible to construct a series of propagators $K^{(n)}$, which converge to a propagator $K^{(\infty)}$ which avoids the problems above and which is the quantum analogue of the perfect action. This is done by performing the quantum analogue of the coarse graining procedure described in the last section, and closely resembles the construction of a Wilsonian renormalization group flow:

For a given discretization $2 N$ one sets up the propagator (3.9), and performs the integration over every odd $t_{2 k+1}, q_{2 k+1}$, keeping the even $t_{2 k}, q_{2 k}$ fixed. For $N=1$ this simply reads

$$
K^{(n+1)}\left(t_{0}, q_{0}, t_{2}, q_{2}\right):=\int_{\mathbb{R}^{2}} d t_{1}, d q_{1} K^{(n)}\left(t_{0}, q_{0}, t_{1}, q_{1}\right) K^{(n)}\left(t_{1}, q_{1}, t_{2}, q_{2}\right) .
$$

This procedure has been tested for the harmonic oscillator ${ }^{8}$ and for the quartic anharmonic oscillator (to linear order in the interaction parameter $\lambda$ ) in [58]. It could be shown that in the limit $n \rightarrow \infty$ the $K^{(n)}$ converge to an infinite contribution coming from the $t_{n}$ integration, times a propagator $K^{(\infty)}$.

The perfect propagator $K^{(\infty)}$ satisfies some crucial properties:

- $K^{(\infty)}$ exhibits the correct gauge symmetry, i.e. it is invariant under a simultaneous transformation of e.g. $t_{0}$ and $q_{0}$ via

$$
t_{0} \rightarrow \tilde{t}_{0}=t_{0}+\Delta t_{0}, \quad q_{0} \rightarrow \tilde{q}_{0}=q_{0}+\Delta q\left(t_{0}, q_{0}, \Delta t_{0}\right),
$$

which are such that $\left(\tilde{t}_{0}, \tilde{q}_{0}\right)$ lie on a solution to the continuum equations of motion connecting $\left(t_{0}, q_{0}\right)$ and $\left(t_{1}, q_{1}\right)$.

- Equivalently, $K^{(\infty)}$ satisfies the correct constraint equation, i.e.

$$
\left(i \hbar \frac{\partial}{\partial t_{0}}-H\left(q_{0}, \frac{\partial}{\partial q_{0}}\right)\right) K^{(\infty)}\left(t_{0}, q_{0}, t_{1}, q_{1}\right)=0,
$$

where $H(q, p)$ is the Hamiltonian of the system.

- $K^{(\infty)}$ is a fixed point of the recursion relation (3.10), if one neglects the $t_{1}$-integration. Due to the symmetry (3.11) it is clear that the integral over $t_{1}$ leads to a divergent result, and this is exactly the volume of the orbit of the discrete gauge symmetry.

- Parametrizing the propagator by $K^{(n)}=\eta^{(n)} e^{\frac{i}{\hbar} S^{(n)}}$, i.e. an action and a measure factor, the relation (3.10) leads to renormalization group equations for $\eta^{(n)}$ and $S^{(n)}$. One can show that in each case at the fixed point $S^{(\infty)}$ agrees with the perfect action for the system, and $\eta^{(\infty)}$ can be regarded as the perfect path integral measure, which contains the quantum corrections to the perfect action, leading to the correct symmetries of $K^{(\infty)}$ reflected in (3.11).

- The quantum theory defined by $K^{(\infty)}$ is discretization-independent, in the sense that it does not depend on $N$, i.e. performing the same calculation for any other $N$ leads back to the same propagator $K^{(\infty)}$.

\footnotetext{
${ }^{7}$ This system has also bee investigated in [55], where the monotonicity of the $t_{n}$ was enforced, i.e. it was only integrated over $t_{0}<\cdots<t_{k}<t_{k+1}<\cdots<t_{N}$, which improves convergence properties. In [33] the integration range for each $t_{k}$ was the whole real line however, since this resembles more closely what happens in e.g. the Ponzano-Regge model [61, 62] where a similar restriction would be very difficult to implement.

${ }^{8}$ Note that due to the discretization, this system is not trivial, since the action is non-polynomial in the $t_{n}$.
} 
It is not hard to see that the last property is a necessary result of the invariance under gauge symmetries (3.11): Assume there is a propagator $K_{N}\left(t_{i}, q_{i}, t_{f}, q_{f}\right)$, which for $N=1$ time steps satisfies an invariance property such as (3.11) for both $\left(t_{i}, q_{i}\right)$ and $\left(t_{f}, q_{f}\right)$. Then the propagator for $N=2$ time steps is given by

$$
K_{2}\left(t_{i}, q_{i}, t_{f}, q_{f}\right)=\int_{\mathbb{R}} d q K_{1}\left(t_{i}, q_{i}, t, q\right) K_{1}\left(t, q, t_{f}, q_{f}\right)
$$

where the integration over $t$ has been dropped since it is effectively the integration over the gauge orbit. By the property (3.11) for $K_{1}$, the right hand side of (3.12) does not depend in $t$. Therefore we can let it go to one boundary value, i.e. $t \rightarrow t_{i}$. But if the dynamics is to be consistent, the propagator $K_{N}$ has to satisfy

$$
K_{N}\left(t, q_{i}, t, q_{f}\right)=\delta\left(q_{i}-q_{f}\right),
$$

which, together with (3.12), results in $K_{1} \equiv K_{2}$. By induction one can easily show that the propagators for any number $N$ of time step $K_{N}$ is equal to $K_{1}$. In this sense, $K_{N}$ is discretizationindependent, and one can always set $N=1$.

\subsection{Renormalization}

The analysis of systems in which the discretization breaks the continuum diffeomorphism symmetry suggests that renormalization methods play a crucial role in any attempt of regaining it. In particular, in the examples observed above, it is only at the RG fixed point where diffeomorphism symmetry is restored in the discrete system, such that the correct number of degrees of freedom is quantized.

This is different from the situation encountered in usual lattice gauge theories, and is due to the fact that the way in which the gauge symmetry and the dynamics interact differently in this case. In lattice gauge theories, one has discretized the system in a way that preserves the gauge symmetries, and there is a good control over the gauge invariant observables (i.e. Wilson loops). Real space renormalization is being carried out in order to find the UV completion of the theory, i.e. incorporate all degrees of freedom by performing the continuum limit. The method of perfect actions is also applied here, but is usually performed to minimize lattice artifacts, and in order to restore the space-time symmetries which have been broken by the lattice, e.g. rotations. Also, they are a tool in order to improve the convergence to the continuum limit when taking the lattice spacing $a \rightarrow 0$ [56].

In the case of GR however, the gauge symmetry and the dynamics are intimately interwined. Being on the gauge-invariant sector of the theory implies being on the solution space of GR as well. Hence a discrete approximation will only have exact gauge symmetry if it contains all the information of the continuum theory, and therefore sits at the RG fixed point.

This adds another point of view to the issue of renormalization in lattice theories to the case of discrete gravity. On the one hand, the RG flow can just be seen as a method of investigating the behavior of a theory described by a (fundamental) lattice model, on different scales. In particular, one can ask under which circumstances the UV limit of the theory describes GR, i.e. in which sense diffeomorphism symmetry emerges. In this case, one does not necessarily have to demand diffeomorphism invariance of the discrete theory. As a result, the discrete theory will contain information about more degrees of freedom than just pure GR. These pseudodegrees of freedom arise in the same way in which e.g. in electromagnetism breaking of the gauge symmetries would result in the emergence of longitudinal photons. In this framework, the main contribution of the path integral would come from triangulations with a large number of vertices, which would render the expansion into vertex numbers problematic [54]. 
From this point of view, the investigation of Spin Foam renormalization deals with the question of how the pseudo degrees of freedom decouple in the thermodynamic limit $^{9}$.

On the other hand, if one were to strictly demand the correct implementation of diffeomorphism symmetry at all scales, then one would have to demand the gauge symmetry to be present on the discrete level (i.e. on a triangulation). One would then have to construct the path integral measures with the correct symmetries, and the analysis of the last sections suggest that renormalization techniques are a helpful tool to achieve this. Only in this case would the corresponding canonical theory support the constraints satisfying the Dirac algebra, exactly also for coarse triangulations. In particular, the theory would most likely be independent under Pachner moves, as has been argued in [65, 66].

Both points of view are physically quite different, and the resulting theories for either case would only agree for large triangulations. The investigation of both, however, require the use of renormalization group techniques, albeit for different purposes.

To investigate these issues, a collection of toy models has been introduced in [67], where the gauge groups within spin foam models are replaced by finite groups, making them more accessible to numerical studies and avoiding many issues with divergencies. In [60] the coarse graining methods described above have been applied to a class of so-called cut-off models based on the Abelian group $\mathbb{Z}_{p}$. In order to deal with the non-local terms which are prevalent in real space renormalization approaches, a Migdal-Kadanoff-type approximation scheme has been employed [59]. Already in $3 D$ one can see that it is a highly nontrivial issue whether the discrete model flows to a fixed point at which diffeomorphism symmetry is realized. The behavior of RG trajectories depends highly on the starting point, leading to a structured phase diagram with several different fixed points.

\section{Summary}

In this article, we have reviewed various angles of the problems that arise in discretizing theories with diffeomorphism symmetry, with (quantum) general relativity in mind.

On the one side, we have discussed in Section 2 how the constraint algebra can be deformed, and how this can lead to all sorts of undesirable problems, such as Lagrange multipliers becoming complex, and one essentially losing control over the quality of the approximation that the discrete theory provides for the continuum theory. To remedy this problem, the uniform discretization approach was presented, which can be seen as a concrete implementation of the master constraint programme. Although the symmetries are not restored on the discrete level, one has good control over the value of the constraints, which can be made arbitrarily small, and constant over time. We have treated two examples, one with a good continuum limit, and another one with a fundamental level of discreteness, in which the continuum theory is only approximated well in some sense. It is widely believed that this should be a characteristic of a quantum theory of gravity.

On the other side, in Section 3 we have demonstrated in which sense the diffeomorphism symmetry is broken in the covariant setting, in particular in Regge Calculus. We have described how one might hope to restore diffeo symmetry on the discrete level, by replacing the Regge action with a so-called perfect action, which can be defined by an iterative coarse-graining process. On the quantum side, this process resembles a Wilsonian renormalization group flow, and it has been shown how, with this method, one can construct both the classical discrete action, as well was the quantum mechanical propagator, with the correct implementation of

\footnotetext{
${ }^{9}$ Note that this is also connected to the question of whether in this limit the path integral is dominated by states which resemble smooth manifolds. This is a long-standing question also in other approaches to discrete gravity such as causal dynamical triangulations [63], and is also under intensive investigation in the GFT approach to spin foams [64].
} 
diffeomorphism symmetry, in certain mechanical toy models, as well as for $3 d$ GR. Whether a similar construction works in $4 d$ general relativity is still an open question, being related to issues of locality and renormalizability, which are still largely open in this context.

\section{References}

[1] Wald R.M., General relativity, University of Chicago Press, Chicago, IL, 1984.

[2] Hojman S.A., Kuchar K., Teitelboim C., Geometrodynamics regained, Ann. Physics 96 (1976), 88-135.

[3] Bergmann P.G., Komar A., The coordinate group symmetries of general relativity, Internat. J. Theoret. Phys. 5 (1972), 15-28.

[4] Hartle J.B., Hawking S.W., Wave function of the universe, Phys. Rev. D 28 (1983), 2960-2975.

[5] Dirac P.A.M., The Theory of gravitation in Hamiltonian form, Proc. Roy. Soc. London. Ser. A 246 (1958), 333-343.

[6] DeWitt B.S., Quantum theory of gravity. I. The canonical theory, Phys. Rev. 160 (1967), 1113-1148.

[7] Loll R., Discrete approaches to quantum gravity in four dimensions, Living Rev. Relativity 1 (1998), 13, 53 pages, gr-qc/9805049.

[8] Ambjørn J., Jurkiewicz J., Loll R., Causal dynamical triangulations and the quest for quantum gravity, in Foundations of Space and Time: Reflections on Quantum Gravity, Cambridge University Press, Cambridge, 2011, 1-19, arXiv:1004.0352.

[9] Dowker F., Causal sets as discrete spacetime, Contemp. Phys. 47 (2006), 1-9. Dowker F., Causal sets and discrete spacetime, AIP Conf. Proc. 861 (2006), 79-88.

[10] Konopka T., Markopoulou F., Smolin L., Quantum graphity, hep-th/0611197.

[11] Ashtekar A., Lewandowski J., Marolf D., Mourao J., Thiemann T., Quantization of diffeomorphism invariant theories of connections with local degrees of freedom, J. Math. Phys. 36 (1995), 6456-6493, gr-qc/9504018. Thiemann T., Modern canonical quantum general relativity, gr-qc/0110034.

Rovelli C., Quantum gravity, Cambridge Monographs on Mathematical Physics, Cambridge University Press, Cambridge, 2004.

[12] Perez A., Spin foam models for quantum gravity, Classical Quantum Gravity 20 (2003), R43-R104, gr-qc/0301113.

Perez A., Topological QFT and spin foams, Living Rev., to appear.

[13] Rovelli C., A new look at loop quantum gravity, Classical Quantum Gravity 28 (2011), 114005, 24 pages, arXiv:1004.1780.

[14] Rovelli C., Smolin L., Spin networks and quantum gravity, Phys. Rev. D 52 (1995), 5743-5759. gr-qc/9505006.

[15] Piran T., Williams R.M., Three-plus-one formulation of Regge calculus, Phys. Rev. D 33 (1986), 1622-1633. Friedman J.L., Jack I., $(3+1)$ Regge calculus with conserved momentum and Hamiltonian constraints, J. Math. Phys. 27 (1986), 2973-2986.

Loll R., On the diffeomorphism commutators of lattice quantum gravity, Classical Quantum Gravity 15 (1998), 799-809, gr-qc/9708025.

[16] Thiemann T., Quantum spin dynamics (QSD), Classical Quantum Gravity 15 (1998), 839-873, gr-qc/9606089.

Thiemann T., Quantum spin dynamics (QSD). II. The kernel of the Wheeler-DeWitt constraint operator, Classical Quantum Gravity 15 (1998), 875-905, gr-qc/9606090.

[17] Lewandowski J., Marolf D., Loop constraints: A habitat and their algebra, Internat. J. Modern Phys. D 7 (1998), 299-330, gr-qc/9710016.

Gambini R., Lewandowski J., Marolf D., Pullin J., On the consistency of the constraint algebra in spin network quantum gravity, Internat. J. Modern Phys. D 7 (1998), 97-109, gr-qc/9710018.

[18] Thiemann T., Quantum spin dynamics (QSD). III. Quantum constraint algebra and physical scalar product in quantum general relativity, Classical Quantum Gravity 15 (1998), 1207-1247, gr-qc/9705017.

[19] Perez A., On the regularization ambiguities in loop quantum gravity, Phys. Rev. D 73 (2006), 044007, 18 pages, gr-qc/0509118.

[20] Yee K., Numerical solution of initial boundary value problems involving Maxwell's equations in isotropic media, IEEE Trans. Antennas and Propagation 14 (1966), 302-307. 
[21] Regge T., General relativity without coordinates, Nuovo Cimento 19 (1961), 558-571.

[22] Marsden J.E., West M., Discrete mechanics and variational integrators, Acta Numer. 10 (2001), 357-514.

[23] Bahr B., Dittrich B., Improved and perfect actions in discrete gravity, Phys. Rev. D 80 (2009), 124030, 15 pages, arXiv:0907.4323.

[24] Miller W.A., The geometrodynamic content of the Regge equations as illuminated by the boundary of a boundary principle, Found. Phys. 16 (1986), 143-169.

Hamber H.W., Williams R.M., Gauge invariance in simplicial gravity, Nuclear Phys. B 487 (1997), 345-408, hep-th/9607153.

Morse P.A., Approximate diffeomorphism invariance in near-flat simplicial geometries, Classical Quantum Gravity 9 (1992), 2489-2504.

Gentle A.P., Kheyfets A., McDonald J.R., Miller W.A., A Kirchoff-like conservation law in Regge calculus, Classical Quantum Gravity 26 (2009), 015005, 11 pages, arXiv:0807.3041.

[25] Dittrich B., Diffeomorphism symmetry in quantum gravity models, Adv. Sci. Lett. 2 (2009), 151-163, arXiv:0810.3594.

[26] Hamber H.W., Williams R.M., Simplicial quantum gravity in three-dimensions: analytical and numerical results, Phys. Rev. D 47 (1993), 510-532.

[27] Rocek M., Williams R.M., Quantum Regge calculus, Phys. Lett. B 104 (1981), 31-37.

Rocek M., Williams R.M., The quantization of Regge calculus, Z. Phys. C 21 (1984), 371-381.

[28] Dittrich B., Freidel L., Speziale S., Linearized dynamics from the 4-simplex Regge action, Phys. Rev. D 76 (2007), 104020, 15 pages, arXiv:0707.4513.

[29] Bahr B., Dittrich B., (Broken) gauge symmetries and constraints in Regge calculus, Classical Quantum Gravity 26 (2009), 225011, 34 pages, arXiv:0905.1670.

Bahr B., Dittrich B., Breaking and restoring of diffeomorphism symmetry in discrete gravity, AIP Conf. Proc. 1196 (2009), 10-17, arXiv:0909.5688.

[30] Conrady F., Freidel L., Semiclassical limit of 4-dimensional spin foam models, Phys. Rev. D 78 (2008), 104023, 18 pages, arXiv:0809.2280.

Barrett J.W., Dowdall R.J., Fairbairn W.J., Gomes H., Hellmann F., Asymptotic analysis of the EnglePereira-Rovelli-Livine four-simplex amplitude, J. Math. Phys. 50 (2009), 112504, 30 pages, arXiv:0902.1170. Barrett J.W., Dowdall R.J., Fairbairn W.J., Gomes H., Hellmann F., Pereira R., Asymptotics of 4d spin foam models, arXiv:1003.1886.

[31] Alexandrov S., Roche P., Critical overview of loops and foams, Phys. Rep. 506 (2011), 41-86, arXiv:1009.4475.

Dittrich B., Ryan J.P., Simplicity in simplicial phase space, Phys. Rev. D 82 (2010), 064026, 19 pages, arXiv:1006.4295.

[32] Dittrich B., Höhn P.A., From covariant to canonical formulations of discrete gravity, Classical Quantum Gravity 27 (2010), 155001, 37 pages, arXiv:0912.1817.

[33] Bahr B., Dittrich B., Steinhaus S., Perfect discretization of reparametrization invariant path integrals, Phys. Rev. D 83 (2011), 105026, 19 pages, arXiv:1101.4775.

[34] Rovelli C., Speziale S., Reconcile Planck-scale discreteness and the Lorentz-Fitzgerald contraction, Phys. Rev. D 67 (2003), 064019, 11 pages, gr-qc/0205108.

[35] Di Bartolo C., Gambini R., Pullin J., Canonical quantization of constrained theories on discrete space-time lattices, Classical Quantum Gravity 19 (2002), 5275-5296, gr-qc/0205123.

Di Bartolo C., Gambini R., Porto R., Pullin J., Dirac-like approach for consistent discretizations of classical constrained theories, J. Math. Phys. 46 (2005), 012901, 14 pages, gr-qc/0405131.

Di Bartolo C., Gambini R., Pullin J., Consistent and mimetic discretizations in general relativity, J. Math. Phys. 46 (2005), 032501, 18 pages, gr-qc/0404052.

Gambini R., Pullin J., Classical and quantum general relativity: a new paradigm, Gen. Relativity Gravitation 37 (2005), 1689-1694, gr-qc/0505052.

Gambini R., Pullin J., Classical and quantum general relativity: a new paradigm, Internat. J. Modern Phys. D 14 (2005), 2355-2360.

Gambini R., Pullin J., Consistent discretizations as a road to quantum gravity, in Approaches to Quantum Gravity, Editor D. Oriti, Cambridge University Press, Cambridge, 2009, 400-414, gr-qc/0512065.

Gambini R., Pullin J., Discrete space-time, in 100 Years of Relativity - Space-Time Structure: Einstein and Beyond, Editor A. Ashtekar, World Scientific, Singapore, 2006, 415-444, gr-qc/0505023.

[36] Jaroszkiewicz G., Norton K., Principles of discrete time mechanics. I. Particle systems, J. Phys. A: Math. Gen. 30 (1997), 3115-3144, hep-th/9703079. 
[37] Gambini R., Pullin J., Discrete quantum gravity: cosmological examples, Classical Quantum Gravity 20 (2003), 3341-3364, gr-qc/0212033.

[38] Gambini R., Ponce M., Pullin J., Consistent discretizations: the Gowdy spacetimes, Phys. Rev. D 72 (2005), 024031, 9 pages, gr-qc/0505043.

[39] Thiemann T., The Phoenix Project: master constraint programme for loop quantum gravity, Classical Quantum Gravity 23 (2006), 2211-2247, gr-qc/0305080.

Thiemann T., Quantum spin dynamics. VIII. The master constraint, Classical Quantum Gravity 23 (2006), 2249-2265, gr-qc/0510011.

Dittrich B., Thiemann T., Testing the master constraint programme for loop quantum gravity. I. General framework, Classical Quantum Gravity 23 (2006), 1025-1065, gr-qc/0411138.

Dittrich B., Thiemann T., Testing the master constraint programme for loop quantum gravity. II. Finitedimensional systems, Classical Quantum Gravity 23 (2006), 1067-1088, gr-qc/0411139.

Dittrich B., Thiemann T., Testing the master constraint programme for loop quantum gravity. III. SL $(2, \mathbb{R})$ models, Classical Quantum Gravity 23 (2006), 1089-1120, gr-qc/0411140.

Dittrich B., Thiemann T., Testing the master constraint programme for loop quantum gravity. IV. Free field theories, Classical Quantum Gravity 23 (2006), 1121-1142, gr-qc/0411141.

Dittrich B., Thiemann T., Testing the master constraint programme for loop quantum gravity. V. Interacting field theories, Classical Quantum Gravity 23 (2006), 1143-1162, gr-qc/0411142.

[40] Campiglia M., Di Bartolo C., Gambini R., Pullin J., Uniform discretizations: a quantization procedure for totally constrained systems including gravity, J. Phys. Conf. Ser. 67 (2007), 012020, 6 pages, gr-qc/0606121. Campiglia M., Di Bartolo C., Gambini R., Pullin J., Uniform discretizations: a new approach for the quantization of totally constrained systems, Phys. Rev. D 74 (2006), 124012, 15 pages, gr-qc/0610023.

[41] Gomberoff A., Marolf D., On group averaging for SO(n,1), Internat. J. Modern Phys. D 8 (1999), 519-535, gr-qc/9902069.

[42] Louko J., Rovelli C., Refined algebraic quantization in the oscillator representation of SL(2, R), J. Math. Phys. 41 (2000), 132-155, gr-qc/9907004.

[43] Gambini R., Pullin J., Rastgoo S., Quantum scalar field in quantum gravity: the vacuum in the spherically symmetric case, Classical Quantum Gravity 26 (2009), 215011, 15 pages, arXiv:0906.1774.

Gambini R., Pullin J., Rastgoo S., Quantum scalar field in quantum gravity: the propagator and Lorentz invariance in the spherically symmetric case, Gen. Relativity Gravitation 43 (2011), 3569-3592, arXiv:1105.0667.

[44] Porter J., A new approach to the Regge calculus. I. Formalism, Classical Quantum Gravity 4 (1987), 375389.

Kheyfets A., LaFave N.J., Miller W.A., Null-strut calculus. I. Kinematics, Phys. Rev. D 41 (1990), 36283636 .

[45] Rivin I., Schlenker J.-M., On the Schlaefli differential formula, math.DG/0001176.

[46] Freidel L., Louapre D., Diffeomorphisms and spin foam models, Nuclear Phys. B 662 (2003), 279-298, gr-qc/0212001.

[47] Piran T., Strominger A., Solutions of the Regge equations, Classical Quantum Gravity 3 (1986), 97-102.

[48] Barrett J.W., Galassi M., Miller W.A., Sorkin R.D., Tuckey P.A., Williams R.M., Paralellizable implicit evolution scheme for Regge calculus, Internat. J. Theoret. Phys. 36 (1997), 815-839, gr-qc/9411008.

[49] Dittrich B., Ryan J.P., Phase space descriptions for simplicial 4D geometries, Classical Quantum Gravity 28 (2011), 065006, 34 pages, arXiv:0807.2806.

[50] Bahr B., Dittrich B., Regge calculus from a new angle, New J. Phys. 12 (2010), 033010, 10 pages, arXiv:0907.4325.

[51] Dittrich B., Höhn P.A., Canonical simplicial gravity, arXiv:1108.1974.

[52] Lehner L., Numerical relativity: a review, Classical Quantum Gravity 18 (2001), R25-R86, gr-qc/0106072.

[53] 't Hooft G., Veltman M.J.G., Regularization and renormalization of gauge fields, Nuclear Phys. B 44 (1972), $189-213$.

[54] Ashtekar A., Campiglia M., Henderson A., Loop quantum cosmology and spin foams, Phys. Lett. B 681 (2009), 347-352, arXiv:0909.4221.

Rovelli C., Vidotto F., On the spinfoam expansion in cosmology, Classical Quantum Gravity 27 (2010), 145005, 10 pages, arXiv:0911.3097.

Henderson A., Rovelli C., Vidotto F., Wilson-Ewing E., Local spinfoam expansion in loop quantum cosmology, Classical Quantum Gravity 28 (2011), 025003, 10 pages. arXiv:1010.0502. 
[55] Rovelli C., Discretizing parametrized systems: the magic of Ditt-invariance, arXiv:1107.2310.

[56] Hasenfratz P., Niedermayer F., Perfect lattice action for asymptotically free theories, Nuclear Phys. B 414 (1994), 785-814, hep-lat/9308004.

Hasenfratz P., Prospects for perfect actions, Nuclear Phys. B Proc. Suppl. 63 (1998), 53-58, hep-lat/9709110.

[57] Bietenholz W., Perfect scalars on the lattice, Internat. J. Modern Phys. A 15 (2000), 3341-3367, hep-lat/9911015.

[58] Bahr B., Dittrich B., He S., Coarse graining theories with gauge symmetries, New J. Phys. 13 (2011), 045009, 34 pages, arXiv:1011.3667.

[59] Migdal A.A., Recursion equations in gauge theories, Sov. Phys. JETP 42 (1975), 413-439.

[60] Dittrich B., Eckert F.C., Martin-Benito M., Coarse graining methods for spin net and spin foam models, arXiv:1109.4927.

[61] Ponzano G., Regge T., Semiclassical limit of Racah coefficients, in Spectroscopic and Group Theoretical Methods in Physics, Editor F. Bloch, John Wiley and Sons, Inc., New York, 1968, 1-58.

[62] Barrett J.W., Naish-Guzman I., The Ponzano-Regge model, Classical Quantum Gravity 26 (2009), 155014, 48 pages, arXiv:0803.3319.

[63] Ambjørn J., Jurkiewicz J., Loll R., Semiclassical universe from first principles, Phys. Lett. B 607 (2005), 205-213, hep-th/0411152.

Ambjørn J., Görlich A., Jurkiewicz J., Loll R., Gizbert-Studnicki J., Trzesniewski T., The semiclassical limit of causal dynamical triangulations, Nuclear Phys. B 849 (2011), 144-165, arXiv:1102.3929.

[64] Bonzom V., Gurau R., Riello A., Rivasseau V., Critical behavior of colored tensor models in the large $N$ limit, Nuclear Phys. B $\mathbf{8 5 3}$ (2011), 174-195, arXiv:1105.3122.

[65] Barrett J.W., Quantum gravity as topological quantum field theory, J. Math. Phys. 36 (1995), 6161-6179, gr-qc/9506070.

[66] Pfeiffer H., Diffeomorphisms from finite triangulations and absence of 'local' degrees of freedom, Phys. Lett. B 591 (2004), 197-201, gr-qc/0312060.

Pfeiffer H., Quantum general relativity and the classification of smooth manifolds, gr-qc/0404088.

[67] Bahr B., Dittrich B., Ryan J.P., Spin foam models with finite groups, arXiv:1103.6264. 